\title{
Uncertainty Quantification of the Traffic Assignment Model
}

\author{
Mundher Seger ${ }^{1,2^{\star}}$, Lajos Kisgyörgy ${ }^{1}$ \\ ${ }^{1}$ Department of Highway and Railway Engineering, Faculty of Civil Engineering, Budapest University of Technology and Economics, \\ 1111 Budapest, Múegyetem rkp. 3. Hungary \\ 2 Civil Engineering Department, University of Technology, Sana'a Street,19006 Baghdad, Iraq \\ * Corresponding author, e-mail: 40123@uotechnology.edu.iq
}

Received: 07 May 2020, Accepted: 11 August 2020, Published online: 16 September 2020

\begin{abstract}
Forecasting of traffic flow in the traffic assignment model suffered to a wide range of uncertainties arising from different sources and exacerbating through sequential-stages of the travel demand model. Uncertainty quantification can provide insights into the level of confidence on the traffic assignment model outputs, and also identify the uncertainties of the input Origin-Destination matrix for enhancing the forecasting robustness of the travel demand model.

In this paper, a systematic framework is proposed to quantify the uncertainties that lie in the Origin-Destination input matrix. Hence, this study mainly focuses on predicting the posterior distributions of uncertainty Origin-Destination pairs and correcting the biases of Origin-Destination pairs by using the inverse uncertainty quantification formulated through Least Squares Adjustment method. The posterior distributions are further used in the forward uncertainty quantification to quantify the forecast uncertainty of the traffic flow on a transport network. The results show the effectiveness of implementing the inverse uncertainty quantification framework in the traffic assignment model. And demonstrate the necessity of including uncertainty quantification of the input Origin-Destination matrix in future work of travel demand modelling.
\end{abstract}

\section{Keywords}

traffic assignment model, origin-destination, forward uncertainty quantification, inverse uncertainty quantification, least-squares adjustment

\section{Introduction}

Traffic assignment is a very critical part of transportation analysis. Traffic assignment models consider reconciling between travel demand and transportation supply to deliver an output, which describes the steady-state of a transportation system [1]. The traffic assignment model usually possesses inherent uncertainties caused by the parametric variability of the input data of the OD matrix $[2,3]$. Propagating these uncertainties through the traffic assignment model on a transport network to the outputs is one aspect of the traffic flow uncertainty [4-6]. Beyond such propagation of OD pairs uncertainties, quantification of the output uncertainty is an essential requisite for the purpose of combine knowledge of uncertainty caused by the model structure with the uncertainty caused by the parametric variability of the OD pairs [7].

In general, the outputs of the traffic assignment models are very requisite for urban and transport planners to decisions related to transport policy and infrastructure expansion. Hence, simple estimates of traffic flow, even if they refer to the most probable values, are not adequate for an adequate and safe assessment due to the risks associated with these decisions. Therefore, these planners have to give insight into the uncertainty margining the traffic assignment forecasts [8]. However, quantification of uncertainties enables the planners to know the possible range of future values for the traffic flows and the probabilities associated with these possible outputs. Accordingly, Quantifying the uncertainty in traffic flows forecasts can lead to more reliable decisions [9].

Uncertainty quantification (UQ) is a technique used to quantify the uncertainty in the model output that arises from the stochastic behavior of the models' parameters. The UQ depends on assigning a distribution of possible values to each model parameter, instead of assuming fixed model parameters as in a deterministic model as illustrated in Fig. 1 [10]. UQ utilized both quantitative models' characterization and reduction of uncertainties in the models' results. UQ widely used in stretching engineering applications and applied mathematics [11]. 
UQ is a multidisciplinary technique with a broad base of applicable methods, including uncertainty propagation, statistical calibration, sensitivity analysis, and inverse analysis; thus, the UQ entered as a crucial ingredient in modelling workflow that aims to provide more practical information for the forecasting [1, 2]. A UQ enables us to model the naturally occurring variation in the input data (i.e. OD matrix) of the traffic assignment model. It also enhances our knowledge about the model by quantifying how the uncertain OD matrix influence on the traffic flow. Additionally, performing a UQ enable the transport planners to compare the outputs' variations of different methods of traffic assignment models. The challenge of UQ comes from the choice of uncertain input distributions: hence, it is necessary to advance our knowledge on prior distributions and justify the choice of them. This approach is usually referred to as inverse uncertainty quantification (Inverse-UQ).

Inverse-UQ aims to estimate the statistical parameters of uncertain inputs based on given observed data [3]. It is similar to parameter calibration in that both of them use the Bayesian inference theory to explore the posterior distributions using numerical techniques [4]. However, unlike parameter calibration, Inverse-UQ also captures the uncertainty in its estimates rather than only determining point estimates of the best-fit input parameters [5]. In this paper, inverse methods based on Bayes' theorem has been used to characterize and estimate the statistical parameters of the input OD matrix of the traffic assignment model. Fig. 2 shows the difference between the UQ and Inverse-UQ.

(A) Traditional deterministic approach

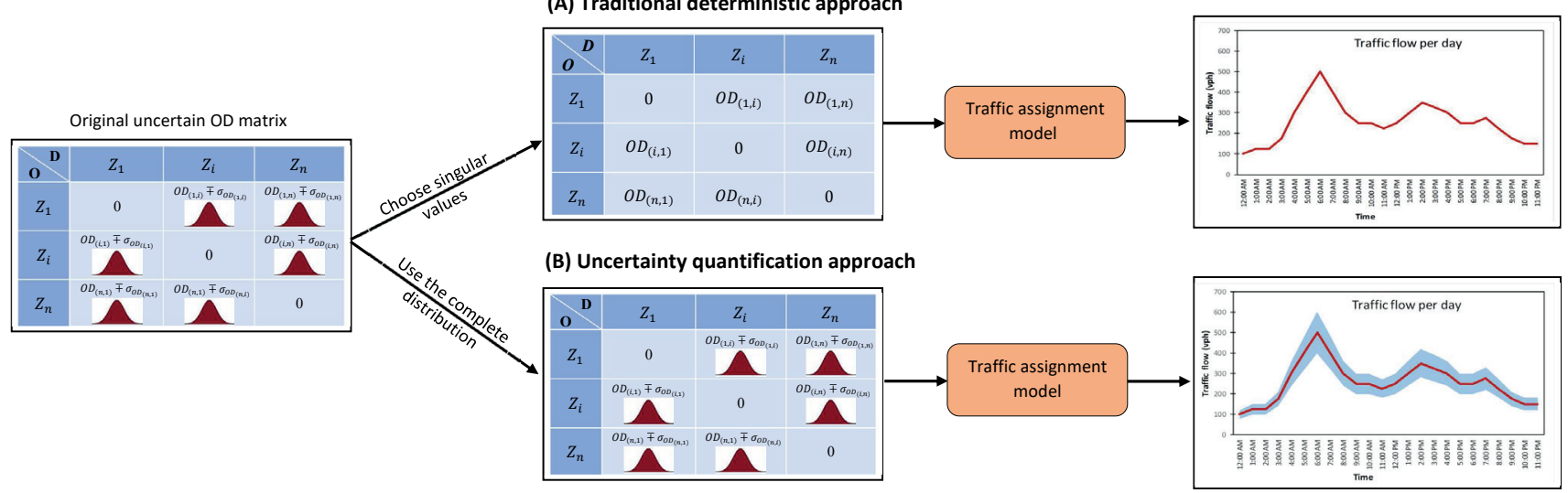

Fig. 1 Illustration of uncertainty quantification of the traffic assignment model; (A) A traditional deterministic approach where each input variable has a chosen fixed value and the output of the model will be an attribute of single values; (B) uncertainty quantification approach of the traffic assignment model takes the distributions of the input variables into account, and the output of the model becomes a range of possible values

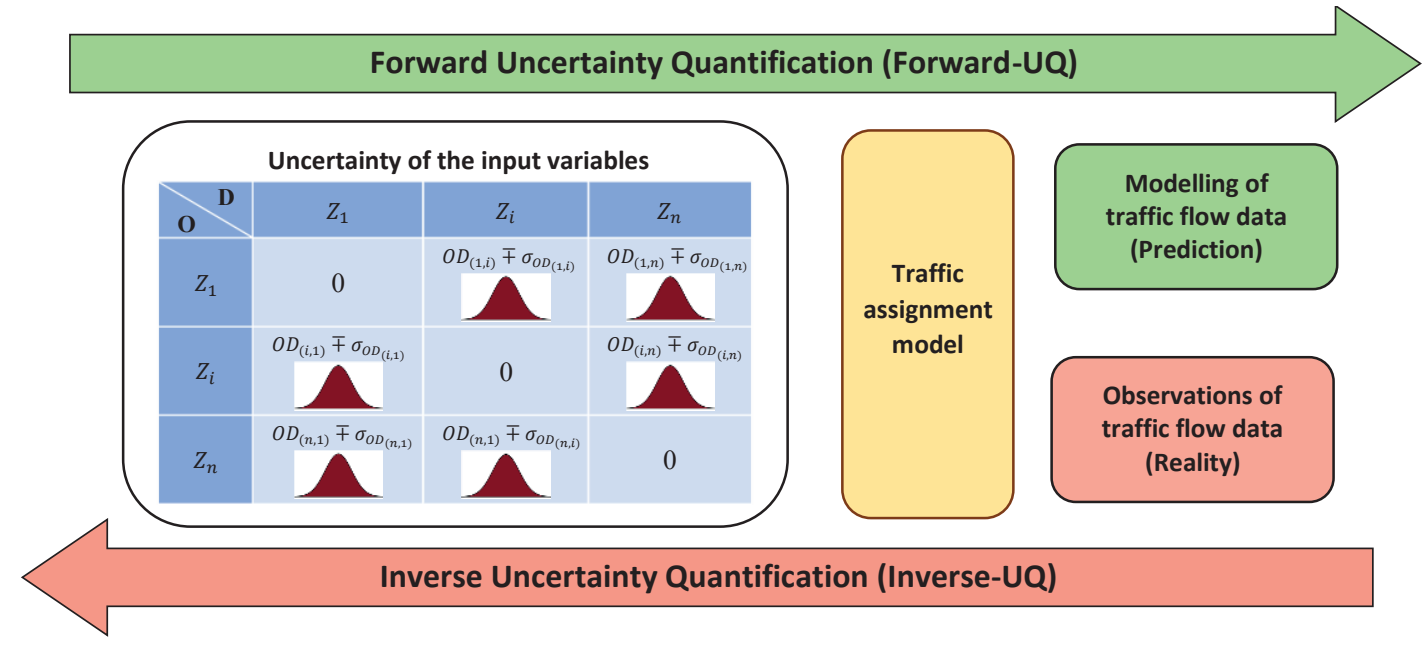

Fig. 2 Illustration the difference between the Forward Uncertainty Quantification and the Inverse Uncertainty Quantification. The Forward Uncertainty Quantification approach of the traffic assignment model propagates the input uncertainties through the model to predicate the statistical parameters of the traffic flow data. The Inverse Uncertainty Quantification approach of the traffic assignment model characterizes the input uncertainties to predicate the statistical parameters of the input variables 
Inverse-UQ technique has gained growing attention in the field of modelling and simulation of the scientific problems, especially in the context of source estimation and calibration of model input parameters or variables [6]. The main object of Inverse-UQ; firstly, is to address the lack of input uncertainty information issues and seek statistical descriptions of the input variables. Secondly, proper identification of the statistical parameters for the input variables also yields an accurate estimation of model discrepancy outputs, which assists in discovering model deficiencies and provides guidance for future improvement of the modelling process. Thirdly provides a better knowledge of the calibrated parameters to produce more reliable model predictions over a comprehensive domain of application [7, 8]. Given the fact that the exact solution of Inverse-UQ is very complex does not exist; therefore, numerical solutions usually employed to estimate the statistical space of the input parameters or variables $[3,9]$. Thus, for this study, the Least Square adjustment method has been used to calibrate the variables of the OD matrix of the traffic assignment model.

Although many of the scientific articles handled the subject of uncertainty in transport models, the literature on quantifying uncertainty in the form of variances, standard deviations, confidence intervals, or other related measures is relatively limited [10]. Furthermore, the researchers who considered the quantification traffic forecasts focused on parameters of the model structure or inputs travel time and trip cost such as; Ashley [11], Lowe et al. [12], Kroes [13], Leurent [14, 15], de Jong et al. [16], Zhang and $\mathrm{Pu}$ [17], Perrakis et al. [18], Deng et al. [19], etc. While the problem of the posterior distribution of the input data of the traffic assignment model did not subject to extensive studies to know the parametric variability of OD pairs.

The importance of this study lies in the obvious need to know the certainty and reliability of traffic forecast results. The input data represented by the OD matrix is one of the most significant sources of variation. Therefore, knowing the parametric variability of input OD pairs enables us to quantify the variability of traffic flow forecasting.

In this study, a new methodology is developed to explore posterior distributions of the input data (i.e. OD matrix) of a traffic assignment model by applying the inverse uncertainty quantification (Inverse-UQ) technique. The key contributions of this methodology present: (1) Calibrating the mean values of the OD pairs to reproduce the average-OD matrix. (2) Estimating the variance values of the OD pairs to create the variance-OD matrix. (3) Quantifying the uncertainty of the traffic assignment model using both the average-OD matrix and variance-OD matrix.

This paper is outlined as follows: Section 2 introduces a brief review of the mathematical background of inverse uncertainty quantification Inverse-UQ problem and least square adjustment LSA technique. Section 3 provides a detailed description of the methodology used to determine the statistical parameters of posterior distributions of the OD pairs. Section 4 shows the application results of this methodology in a study area and apply the results to quantify the uncertainty of the traffic assignment model for the study area, followed by conclusions in Section 5 .

\section{Background}

\subsection{Forward uncertainty quantification}

Explicit quantification of data and structural uncertainties in conceptual modelling is a major scientific and engineering challenge. In any modelling endeavor, reducing the total predictive uncertainty requires a robust quantitative understanding of each of its sources [20]. Assessment of uncertainties is about covering all possible outcomes and their likelihoods, not just the "most likely" outcome. Probabilistic descriptions of model inputs make it possible to derive probability distributions of model outputs and system performance indices. A quantitative approach to uncertainty analysis, where probability distribution functions are assigned to the uncertain variables, is the most appropriate way to deal with uncertainty [21].

Forward Uncertainty quantification (Forward-UQ) is the process of representing uncertainties in system inputs and parameters, then propagating the uncertainty through the model to predict the overall uncertainty in the model results. Forward-UQ tries to determine how likely specific outputs are if some aspects of the system are not precisely known. Moreover, Forward-UQ describes the stochastic behaviour of outputs of interest due to uncertain inputs [1, 22, 23].

Procedures of Forward-UQ can generally be categorized as intrusive or non-intrusive. Intrusive Forward-UQ method requires reformulating the mathematical equations of the models describing the real system processes. Non-intrusive Forward-UQ method, on the other hand, use sets of simulation techniques where simulation elements ensemble is generated by sampling the uncertain inputs according to different sampling schemes. The impact of the input uncertainties can then be analyzed for the chosen model output quantities of interest [24, 25]. 
This study will focus on the intrusive Forward-UQ method; this method requires to know the parametric variability of the input variables that describe statistics of uncertainty. The errors propagation technique widely used for the Forward-UQ problems, the basic formula for errors propagation technique as follows [26, 27]:

Let consider a model with a mathematical function:

$y=f\left(x_{1}, x_{2}, \ldots, x_{n}\right)$,

where: $y$ is the model output, $x_{n}$ being a vector of $n$ input stochastic variables.

Each variable has an uncertainty represents by variance $\sigma_{x}^{2}$. So, the mathematical function with uncertainties will be as follows:

$y+\sigma_{y}^{2}=f\left(\left(x_{1}+\sigma_{x_{1}}^{2}\right),\left(x_{2}+\sigma_{x_{2}}^{2}\right), \ldots,\left(x_{n}+\sigma_{x_{n}}^{2}\right)\right)$,

where $\sigma_{y}^{2}$ is the model uncertainty which, represents the variance in the model output.

The output variance $\sigma_{y}^{2}$, with respect to the variances in variables $x_{1}, x_{2}, \ldots, x_{n}$ can be approximated using partial derivatives.

$\sigma_{y}^{2} \simeq \sigma_{x_{1}}^{2}\left(\frac{\partial y}{\partial x_{1}}\right)^{2}+\sigma_{x_{1}}^{2}\left(\frac{\partial y}{\partial x_{2}}\right)^{2}+\ldots+\sigma_{x_{1}}^{2}\left(\frac{\partial y}{\partial x_{n}}\right)^{2}$

This function can be applied under all conditions and can be used straight once each partial derivative is found and mathematically evaluated $[6,27]$.

\subsection{Inverse uncertainty quantification}

Inverse analysis can be used in the solution of uncertainty quantification problem in the mathematical and engineering sciences, because of the ability to solve challenges of the interactions between analysis, computation, probability, and statistics and the applicability in various complex mathematical models. In general, analysis of the inverse problems confronts mathematical models with observed data to derive the inputs needed to run the models; information of these inputs can then be used to develop control strategies based on forecasts. Both model and input data are usually suffering to uncertainty, as are the resulting conclusions and forecasts; as a result, any decisions or control strategies based on predictions will be significantly improved if quantitative uncertainty is confirmed. In this context, the inverse uncertainty quantification (Inverse-UQ) is the process to quantify the uncertainties of input parameters based on observed outputs data [3, 28, 29].
Inverse-UQ is widely used during the last decades for complex mathematical computerized models to calibrate the uncertainty input parameters or variables by using statistical inference between the results of the computations and the observed data. Ordinarily, the calibration process categorized into two types deterministic calibration and statistical calibration also called the Bayesian approach. The deterministic calibration determines the point estimates of best-fit input parameters such that the discrepancies between model output and observed data can be minimized [3,9]. While the statistical calibration or that is used in Inverse-UQ concern to calibrate the parameters or variables under uncertainty produces statistical descriptions like distributions. Inverse-UQ adopts the Bayesian inference theory to explore the posterior PDF for the parameters or variables. The advantage of calibration using Inverse-UQ is apparent in: firstly, information from observations is never adequately accurate to enable inference of the precise values of the input parameters or variables. Secondly, quantifying the correlations between the estimates. Finally, the observed data usually holds some degree of uncertainty, which should be considered during the inference process of calibration parameters or variables $[6,28]$.

The Bayesian inference theory used to determine the posterior PDF of the posterior PDF for the parameters or variables of any system model concern in the following compact form:

$$
Y^{\text {obs. }}(X)=Y^{\text {mod. }}(X)+V \text {, }
$$

where $Y^{\text {obs. }}$ is a vector for the observations, $Y^{\text {mod. }}$ is a vector for the system model, $X$ is a vector for the unknown model parameters or variables, and $V$ is a vector for the model uncertainty. The precise calibration parameters or variables $X$ which is defined as $p\left(X \mid Y^{0 b s}, Y^{\text {mod }}\right)$. According to Bayesian inference theory [30]:

$$
p\left(X \mid Y^{\text {obs. }}, Y^{\text {mod. }}\right) \propto p\left(Y^{\text {obs. }}, Y^{\text {mod. }} \mid X\right) \cdot p(X),
$$

where $p(X)$ is the prior PDF of the parameter or variables, and $p\left(Y^{o b s}, Y^{\text {nod }} \mid X\right) \mathrm{v}$ is the likelihood function.

The likelihood equation that quantifies the mismatch between the model outputs $Y^{\text {mod. }}$ and the observations $Y^{\text {obs. }}$ is applied to find the posterior distribution over $X$ :

$$
L\left(X \mid Y^{\text {obs. }}, Y^{\text {mod. }}\right) \equiv p\left(Y^{\text {obs. }}, Y^{\text {mod. }} \mid X\right) \text {. }
$$

When the model uncertainty is assumed to fit Gaussian distribution, the likelihood $L\left(X \mid Y^{\text {obs }}, Y^{\text {mod. }}\right)$ can be expressed as [30]: 


$$
L\left(X \mid Y^{\text {obs. }}, Y^{\text {mod. }}\right)=\frac{1}{\sqrt{2 \pi \sigma^{2}}} \exp \left\{-\frac{1}{2 \sigma^{2}}\left[Y^{\text {obs. }}-Y^{\text {mod. }}\right]^{2}\right\}
$$

where $\sigma^{2}$ is the variance of the errors. Error $\varepsilon$ as independent and identically distributed (i.i.d.) zero-mean Gaussian noise, whose variance is expected to be reported along with observed data. In most situations, analytical forms of $p\left(X \mid Y^{\text {obs. }}, Y^{\text {mod. }}\right)$ do not exist; therefore numerical solutions usually used to explore the input parameters or variables space and estimate $p\left(X \mid Y^{\text {obs. }}, Y^{\text {mod }}\right)$.

\subsection{Least Squares Adjustment}

Least Squares Adjustment (LSA) is a numerical technique employed to solve an overdetermined system of equations based on the principle of the Least Squares (LS) technique to minimizes the sum of the squares of differences between the observation and estimate $[31,32]$. The LS method is the efficient and soundest analytical technique for estimating parameters from a set of data [33]. The parameters determined by this method are normally distributed about the true parameter's value with the least possible standard deviations. The accuracy of an estimated parameter expresses the degree of closeness to its real value. Plus, the precision of the estimated parameter expresses the degree of closeness between the estimated parameter. The precision of adjusted parameters is given usually by the variance or standard deviation [34].

The principle of the LS is the estimation of unknown parameters, given a set of observations, satisfying minimizes the sum of the squares of the residuals [33, 34]. These residuals can be expressed mathematically as:

$\sum_{i=1}^{n} V_{i}^{2}$ is minimum,

where $V_{i}$ is the set of residuals $V_{i}=\sum_{j=1}^{m} v_{j}^{2}$, and $n$ is the number of parameters. $v_{i}$ is expressed as:

$v_{i}=\mathrm{y}_{i}^{o b s .}-\mathrm{y}_{i}^{\text {mod }}$.

The observed values of the model system:

$\mathrm{y}_{i}^{\text {obs. }}=f(X)$,

where $X=x_{1}, x_{2}, \ldots, x_{\mathrm{m}}$ the set of estimated parameters, $m$ is the number of parameters in the model.

And the approximate values of the model system:

$\mathrm{y}_{i}^{\text {mod. }}=f\left(X^{\circ}\right)$,

Where $X^{\circ}=x_{1}^{\circ}, x_{2}^{\circ}, \ldots, x_{m}^{\circ}$ is the set of approximate values of the unknown parameters.
The set of estimated parameters:

$X=X^{\circ}+W \cdot X^{c}$,

where $X^{c}=x_{1}^{c}, x_{2}^{c}, \ldots, x_{m}^{c}$ is the set of the correction to be determined by LSA, and $W=w_{1}, w_{2}, \ldots, w_{m}$ is the weight of the parameters.

The relation between the system model and its residual to the estimated parameters and its residuals is given by:

$\mathrm{y}_{i}^{\text {mod. }}+v_{i}=f\left(X^{\circ}+W X^{c}\right)$.

The function $f\left(X^{\circ}+W X^{c}\right)$ can be fragmented using Taylor's series and truncating at the first order to:

$\mathrm{y}_{i}^{\text {mod. }}+v_{i}=f\left(X^{\circ}\right)+W \frac{\partial f}{\partial X} X^{c}$.

Thus, the relation between the residual system model and the set of the correction is given by:

$v_{i}=W \frac{\partial f}{\partial X} X^{c}$

\section{Methodology}

In this paper, a new methodology is developed to quantify the traffic flow uncertainty of the traffic assignment model. The proposed methodology consists of two sequential processes: The first is the inverse uncertainty quantification. And the second is the forward uncertainty quantification. The Inverse-UQ process aims for discovering the statistical parameters of the posterior distribution of the OD pairs using the Least Squares Adjustment method (LSA). While the Forward-UQ process uses the posterior distribution of the OD pairs to quantify the traffic flow forecasts. Fig. 3 illustrates the methodology processes.

\subsection{Inverse-UQ Process}

For this process, the Inverse-UQ technique has been used to the statistical parameters of the posterior distribution of input OD pairs of traffic assignment models. Inverse-UQ was formulated depending on the LSA method. The Inverse-UQ Process consists of three stages: (i) input stage; (ii) calibration stage; (iii) calculation stage. The relationships connecting these stages of the methodology are presented in Fig. 4, and the mathematical and logical computations of this methodology are illustrated in (Appendix A - Algorithm 1).

\subsubsection{Data stage}

The required data for this stage consists of four main components: Firstly, is collecting traffic flow data $\left(q^{o b s .}\right)$. Secondly, it is defining the base travel demand matrix $\left(O D^{\text {base }}\right)$. Thirdly 

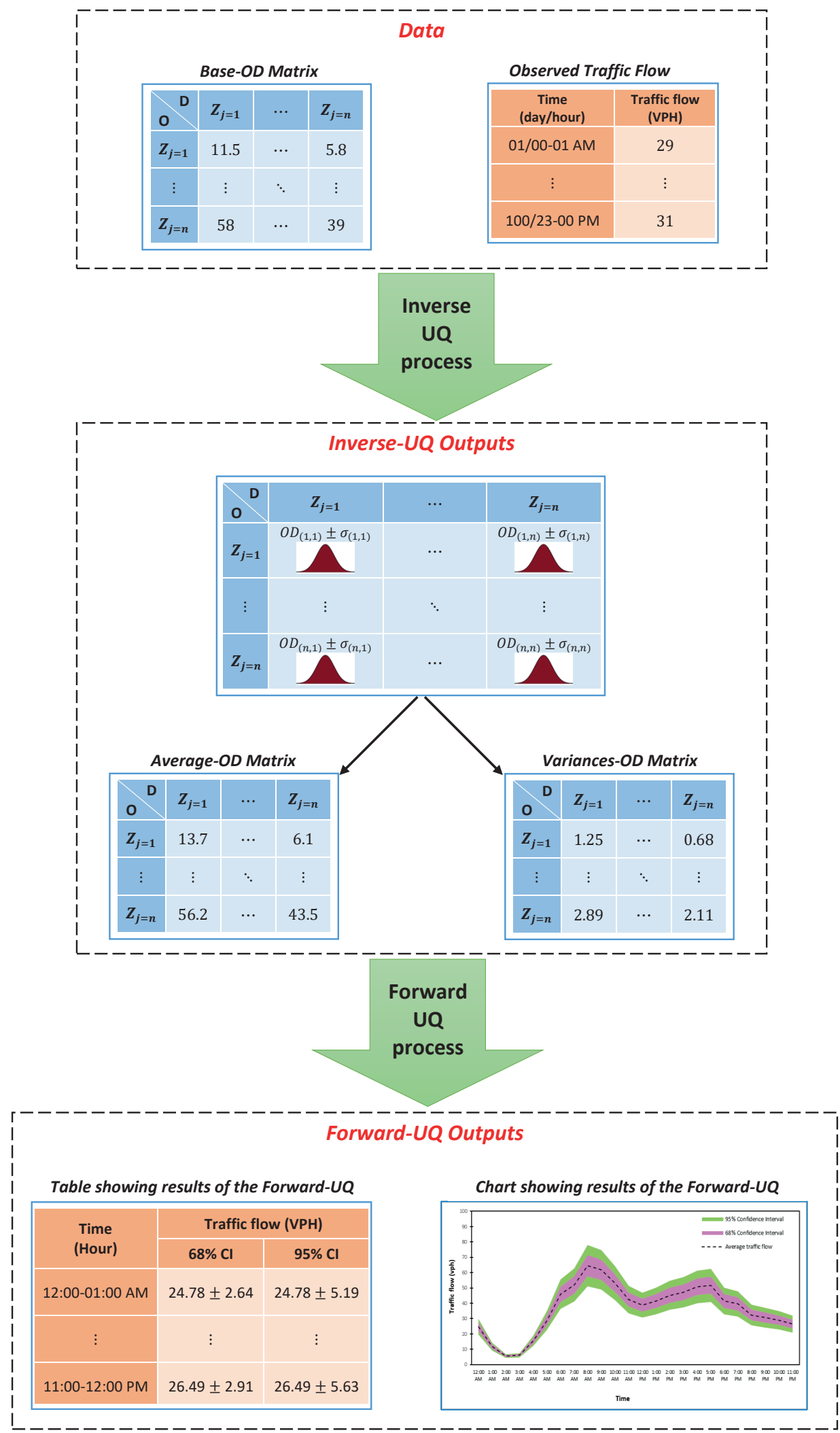

Fig. 3 A flowchart illustrates the methodology process 
is specifying the sample size of observations hours $(N)$. Fourth is establishing a benchmark to define the accuracy limit of the results; So, the Minimum Residual Sum of Squares (Min. RSS) had adopted. Where the RSS is a measure of the difference between the data and an estimation model. A small RSS indicates a close fit of the calibrated OD pairs to the real data.

\subsubsection{Calibration stage}

In this stage, a MATLAB code has been written and linked with the Component Object Model (COM) of VISUM software. This code was executed according to the number of samples $(N)$. Given observed traffic flow $\left(q^{\text {obs }}\right)$, the InverseUQ technique based on LSA has been used to calibrate the input base OD matrix ( $\left.O D^{\text {base }}\right)$. The critical components of the calibration processes are as the following steps:

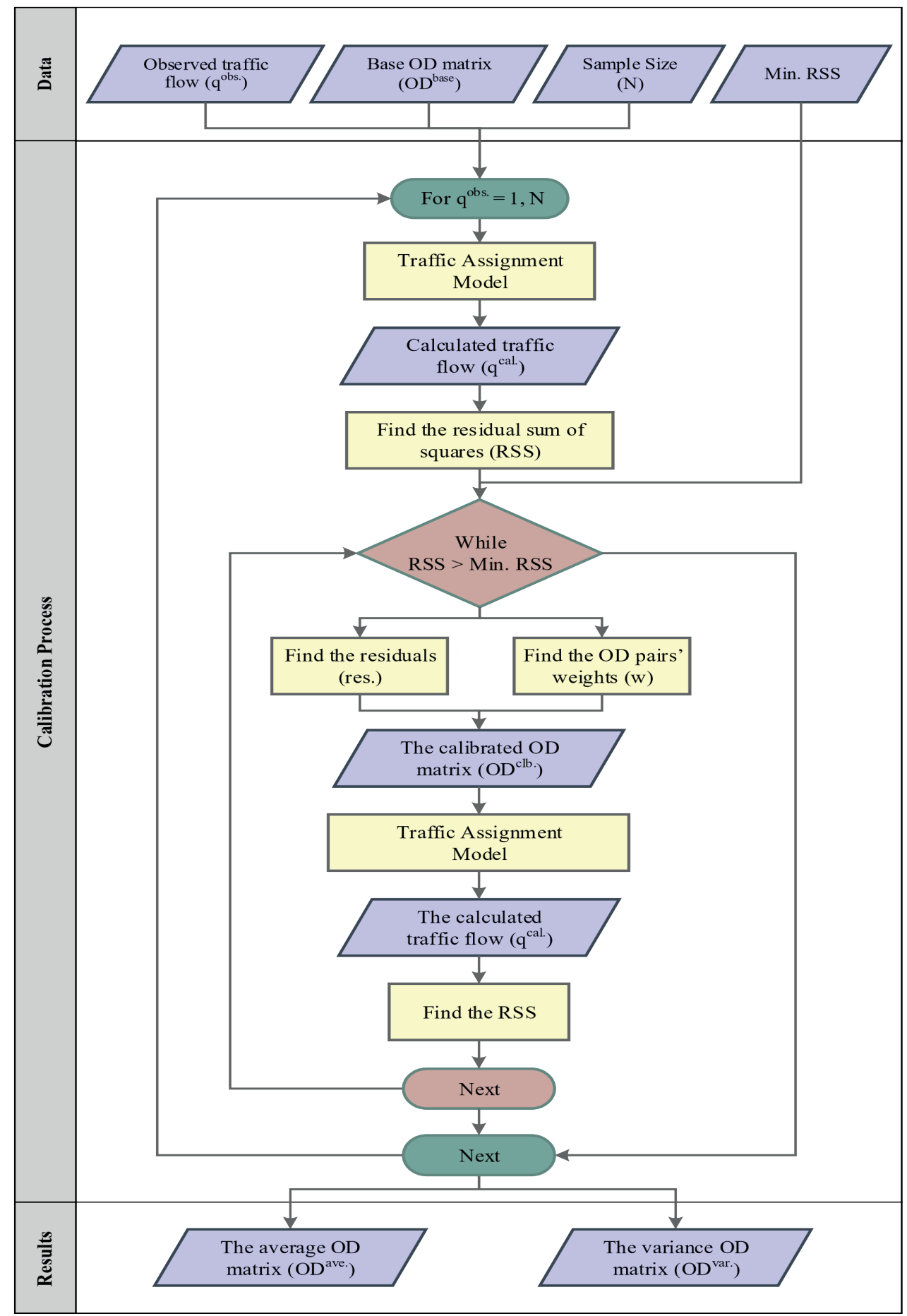

Fig. 4 A flowchart illustrates the methodology stages and the relationship between the stage's components 
- Assume the calibrated OD matrix $\left(O D^{c l b}\right.$ ) equal to base OD matrix $\left(O D^{\text {base }}\right)$ for each observed sample. $O D_{p=1, P}^{c l b}=O D_{p=1, P}^{\text {base }}$

- Finding the calculated traffic flow $\left(O D^{\text {cal. }}\right)$, by using the input base OD matrix $\left(O D^{\text {base }}\right)$ through the traffic assignment model in VISUM software.

- Find the residual sum of squares (RSS).

$$
R S S=\sum_{l=1}^{L}\left(q_{l}^{\text {obs. }}-q_{l}^{c a l .}\right)^{2}
$$

- Compare the RSS with Min.RSS. If RSS less than or equal Min.RSS that means the input OD matrix is accurate; therefore, the calibration process is not required. Otherwise, the input OD matrix needs to calibrate.

- Find the residual for each link on the transport network.

$$
v_{l}=q_{l}^{\text {obs. }}-q_{l}^{c a l} .
$$

Where $v_{l}$ is the residual of the traffic flow for a link $(l)$.

- Find the weight of each OD pair in every link $\left(w_{p}^{l}\right)$.

$$
w_{p}^{l}=\frac{q_{l}^{o b s .}}{\beta_{l}^{*} O D_{p}^{c l b} .}
$$

- Where $\beta_{l}$ is the ink choice probabilities set (i.e. the proportion of the travel demand of the link choice set $\mathcal{F}_{l}$ ).

- Find the correction factor of each OD pair in every link $\left(C_{p}{ }^{l}\right)$, using the LSA method by the partial derivative for each input OD pairs in a set of link choice of the traffic assignment model.

$$
C_{p}^{l}=\frac{v_{l}}{w_{p}^{l}}
$$

- Find the correction factor of each OD pair $\left(C_{p}\right)$.

$$
C_{p}=\frac{\left(\sum_{l=1}^{L} C_{p}^{l}\right)}{L}
$$

- Find the calibrated OD pair $\left(O D_{p}^{c l b}\right)$.

$$
O D_{p}^{c l b .}=O D_{p}^{c l b .}+C_{p}
$$

- Repeat steps 2 to 8.

The calibration process will continue until all the observed samples are performing.

\subsubsection{Calculation stage}

The results of the calibration stages are used to establish the average-OD matrix $\left(O D^{\text {ave }}\right)$ and the variance-OD matrix $\left(O D^{\text {var }}\right)$.
The average-OD matrix:

$O D^{\text {ave. }}=\sum_{p=1}^{P} \frac{\left(\sum_{i=1}^{N} O D_{p}^{c l b .}\right)}{N}$

The variance-OD matrix:

$O D^{v a r .}=\sum_{p=1}^{P} \frac{\left(\sum_{i=1}^{N}\left(O D_{p}^{c l b .}-O D_{p}^{a v e .}\right)^{2}\right)}{N}$

\subsection{Forward-UQ Process}

The obtained average-OD matrix $\left(O D^{\text {ave }}\right)$ and variance-OD matrix $\left(O D^{v a r}\right.$ ) from the Inverse-UQ process will be used in the Forward-UQ process in order to quantify the future traffic flow forecasts. The Forward-UQ process consists of two steps: The first is the reconfiguration of the OD matrix, and the second is the quantification of the traffic flow forecasts. The mathematical computations of this methodology are illustrated in Appendix A-Algorithm 2.

\subsubsection{Reconfiguration the OD matrix}

Both of the average-OD matrix $\left(O D^{\text {ave }}\right)$ and the varianceOD matrix $\left(O D^{v a r}\right)$ are utilized to configure the maximum probable-OD matrix $\left(O D^{\text {max.pob. }}\right)$ and minimum probableOD matrix $\left(O D^{\text {min.pob. }}\right)$ according to different confidence intervals $(C I)$. For this study, two confidence intervals $(C I)$ were adopted: $68 \%$ and $95 \%$. The $C I=68 \%$ comparable to $1.00 \times \sqrt{\sigma^{2}}$, on the other hand, the $C I=95 \%$ comparable to $1.96 \times \sqrt{\sigma^{2}}$.

The max. probable OD matrix within $(68 \% C I)$ :

$$
O D^{\text {max.prob.@ } C I=68 \%}=O D^{\text {ave. }}+1.00 \times \sqrt{O D^{\text {var. }}}
$$

The min. probable OD matrix within $(68 \% C I)$ :

$$
O D^{\text {min.prob.@ } C I=68 \%}=O D^{\text {ave. }}-1.00 \times \sqrt{O D^{\text {var. }}}
$$

The max. probable OD matrix within $(95 \% C I)$ :

$$
O D^{\text {max.prob.@ } C I=95 \%}=O D^{\text {ave. }}+1.96 \times \sqrt{O D^{\text {var }}}
$$

The min. probable OD matrix within $(95 \% C I)$ :

$$
O D^{\text {min.prob. } @ C I=95 \%}=O D^{\text {ave. }}-1.96 \times \sqrt{O D^{\text {var }}} \text {. }
$$

The bias of every base-OD pair:

$\eta=O D^{\text {base }}-O D^{\text {ave }}$

\subsubsection{Quantification the traffic flow forecasts}

Finally, quantifying the traffic flow in each link on the transport network, using the errors propagation theory, by finding the average traffic flow $\left(q_{l}^{\text {ave. }}\right)$ and the probable flow $\left(q_{l}^{\text {prob. }}\right)$. 
The average traffic flow $\left(q_{l}^{\text {ave }}\right)$ :

$q_{l}^{\text {ave. }}=f_{T O \rightarrow D}\left(O D^{\text {ave. }}\right)$.

The max. probable traffic flow value of at $C I=68 \%$ :

$q_{l}^{\text {max.prob.@@CI=68\% }}=f_{T O \rightarrow D}\left(O D^{\text {max.prob.@ } C I=68 \%}\right)$

The min. probable traffic flow value of at $C I=68 \%$ :

$q_{l}^{\text {min.prob.@CI=68\% }}=f_{T O \rightarrow D}\left(O D^{\text {min.prob.@ } C I=68 \%}\right)$

The max. probable traffic flow value of at $C I=95 \%$ :

$q_{l}^{\text {max.prob.@CI=95\% }}=f_{T O \rightarrow D}\left(O D^{\text {max.prob.@ } C I=95 \%}\right)$

The min. probable traffic flow value of at $C I=95 \%$ :

$q_{l}^{\text {min.prob.@ } C I=95 \%}=f_{T O \rightarrow D}\left(O D^{\text {min.prob. } @ C I=95 \%}\right)$

Where $f_{T O \rightarrow D}$ is a traffic assignment model.

Moreover, for the validation purpose of the accuracy of methodology results, the estimated traffic flow by this methodology $\left(q_{l}^{\text {est. }}\right)$ could be compared with the real observed traffic flow $\left(q_{l}^{\text {obs }}\right)$.

The estimated traffic flow $\left(q_{l}^{\text {est }}\right)$ :

$q_{l}^{e s t .}=f_{T O \rightarrow D}\left(O D^{c l b .}\right)$.

\section{Case study}

The proposed methodology was implemented in Ajka town located in Hungary. The Ajka town consists of 25 zones (22 internal zones and 3 external zones), 26 nodes, and 56 links. Fig. 5 shows the transportation network and the traffic analysis zones of Ajka.

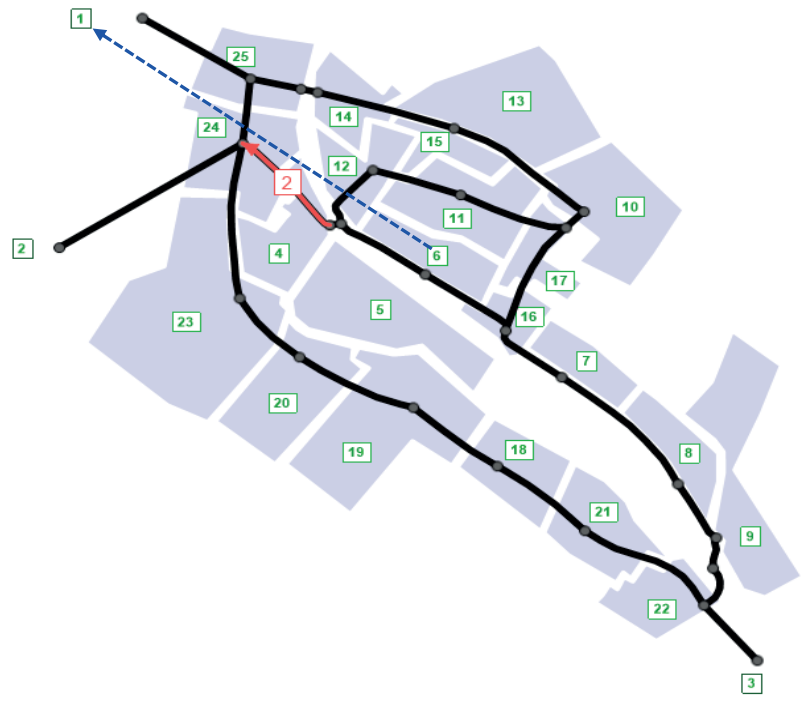

Fig. 5 Study area (Ajka, Hungary), shows the transport network and traffic analysis zones

The inputs data for this study case include base-OD matrix as shown in (Appendix B), the traffic flow observation period continued for 100 days for all transportation links (i.e. sample size $N=100$ ), and the $\min . R S S \leq 1$ was picked. The final result of the calibration process is represented into two matrices; the first is the average-OD matrix $\left(O D^{a v e}\right.$ ) which is created by applying Eq. (22), as shown in (Appendix B), and the second is the variance-OD matrix $\left(O D^{v a r}\right)$ which is created by applying Eq. (23), as shown in (Appendix B). These two matrices providing full information about the probability distributions for all OD pairs by giving the mean $(\mu)$ and the variance $\left(\sigma^{2}\right)$ for each one.

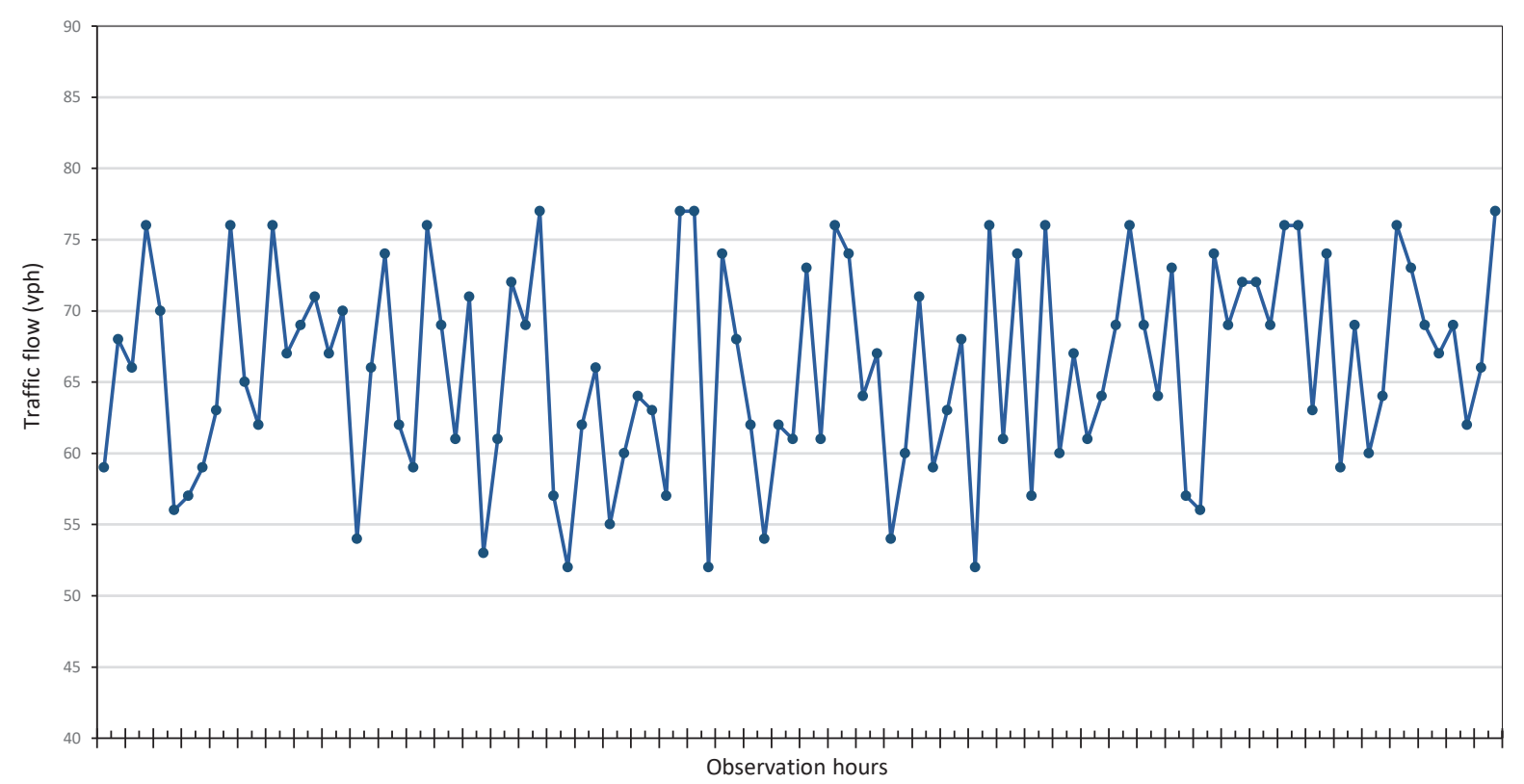

Fig. 6 Traffic flow $\left(q_{02}\right)[\mathrm{vph}]$ at the time (8:00-9:00 AM) during the observed period 
For this paper, the application of the proposed methodology is presented for one link only; the chosen link is $\left(l_{02}\right)$, this link summarises the traffic assignment pattern of other links in this case study. Fig. 6 shows the traffic flow $\left(q_{02}\right)$ at the time (8:00-9:00 AM) during observed periods.

The link choice set of link \#2 $\left(\mathcal{F}_{02}\right)$ has involved 17 OD pairs, and the link choice probabilities set is 1.00 for all pairs.

$$
\begin{aligned}
\mathcal{F}_{02}=\{ & O D_{04-01}, O D_{05-01}, O D_{06-01}, O D_{07-01}, O D_{11-01}, \\
& O D_{12-01}, O D_{16-01}, O D_{04-02}, O D_{06-02}, O D_{07-02}, \\
& O D_{11-02}, O D_{12-02}, O D_{16-02}, O D_{04-03}, O D_{05-03}, \\
& \left.O D_{11-03}, O D_{12-03}\right\}
\end{aligned}
$$

The result of the calibration process for the set $\left(\mathcal{F}_{02}\right)$ illustrated in (Appendix C), which shows the scatter-plots of the calibrated OD pairs $\left(O D^{c l b}\right.$ ) vs. the observed traffic flow $\left(q_{2}^{\text {obs. }}\right)$ surrounded by $68 \%$ and $95 \%$ confidence intervals boundaries, and (Appendix D), which shows probability distributions of the OD pairs of the set. For example, Fig. 7 shows the result of the calibration process for the OD pair from zone 06 to zone $01\left(O D_{06-01}\right)$. The right side shows the probability distribution $\left(O D_{06-01}\right)$, and the left side shows the scatter-plots of the calibrated OD pair $\left(O D_{06-01}^{c l b}\right)$ vs. the observed traffic flow $\left(q_{2}^{\text {obs }}\right)$, surrounded by $68 \%$ and $95 \%$ confidence intervals boundaries.

To provide reliable insight into the accuracy of the calibration process, the observed traffic flow $\left(q_{2}^{o b s}\right)$ with the estimated traffic flow $\left(q_{2}^{\text {est }}\right)$ was compared by the traffic assignment model via VISUM software using the calibrated OD matrix $\left(O D^{c l b}\right.$ ). The comparison was repeated 100 times, according to the number of observations $(N)$. Fig. 8 presents the comparison result, the right-side

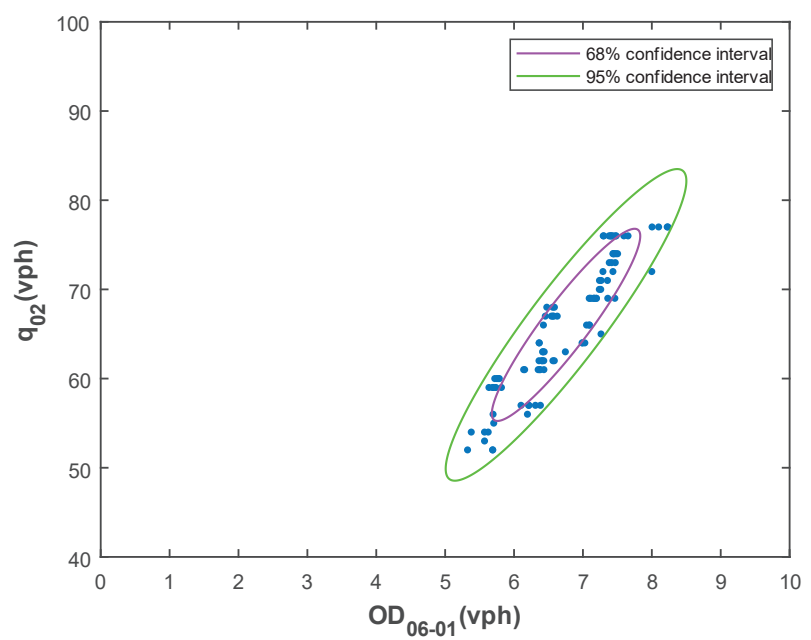

showing the probability distributions of both observed traffic flow $\left(q_{2}^{o b s}\right)$ and estimated traffic flow $\left(q_{2}^{\text {est }}\right)$, a significant matching between them can be seen. Moreover, the right-side indicating the cross-validation of observed traffic flow $\left(q_{2}^{o b s}\right)$ and estimated traffic flow $\left(q_{2}^{\text {est }}\right)$, which gives $R^{2}=0.9994$.

For the sake of simplicity, the statistical parameters of the $\mathcal{F}_{02}$ set with their analytical conclusions are illustrated in Table 1, which includes the base-OD pairs $\left(O D^{\text {base }}\right)$, the distribution parameters of OD pairs: average-OD pairs $\left(O D^{\text {ave. }}\right)$ and variances-OD pairs $\left(O D^{v a r}\right)$. As well as, bias () calculations of the base-OD pairs $\left(O D^{\text {base }}\right)$ compared to the average-OD pairs $\left(O D^{\text {ave }}\right)$, and the probable-OD pairs $\left(O D^{\text {prob. }}\right)$, under two confidence intervals $68 \%$ and $95 \%$ respectively. In this case study, it can be noticed that the most base-OD pairs $\left(O D^{\text {base }}\right)$ were having a negative bias from the average-OD values $\left(O D^{a v e}\right)$, except $O D_{11-01}$ and $O D_{11-03}$ which they had a positive bias.

Finally, the traffic flow uncertainty was quantified by including the variations of the OD pairs of the $\operatorname{set}\left(\mathcal{F}_{02}\right)$, by finding the probable traffic flow $\left(q_{2}^{\text {prob. }}\right)$ in two confidence intervals ( $68 \%$ and $95 \%$ ) for 24 hours. The results of the UQ were illustrated with details in Fig. 9 and Table 2.

\section{Conclusions}

This paper introduces a practical methodology based on the UQ framework for quantifying the traffic flow uncertainty of a traffic assignment model. The main aim of this paper is to explorer the uncertainties of the OD matrix and estimate their parameters, which cause a variation and uncertainty in the traffic flow of the traffic assignment model. The framework implemented in this methodology

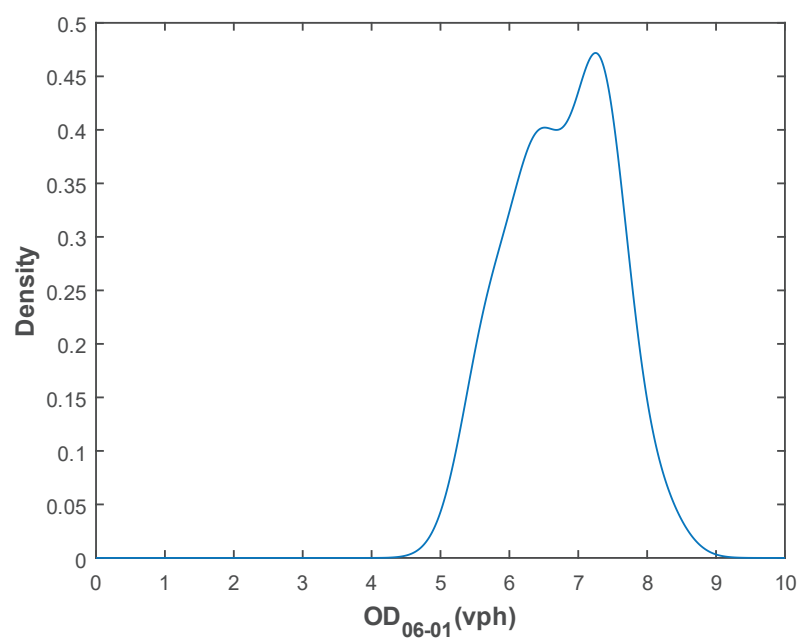

Fig. 7 shows the result of the calibration process for the $\left(O D_{06-01}\right)$. The right side shows the probability distribution, and the left side shows the scatterplots of the calibrated OD pair $\left(O D_{06-01}^{\text {clb. }}\right)$ vs. the observed traffic flow $\left(q_{2}^{\text {obs. }}\right)$ surrounded by the $(68 \%$ and $95 \%)$ confidence intervals boundaries 

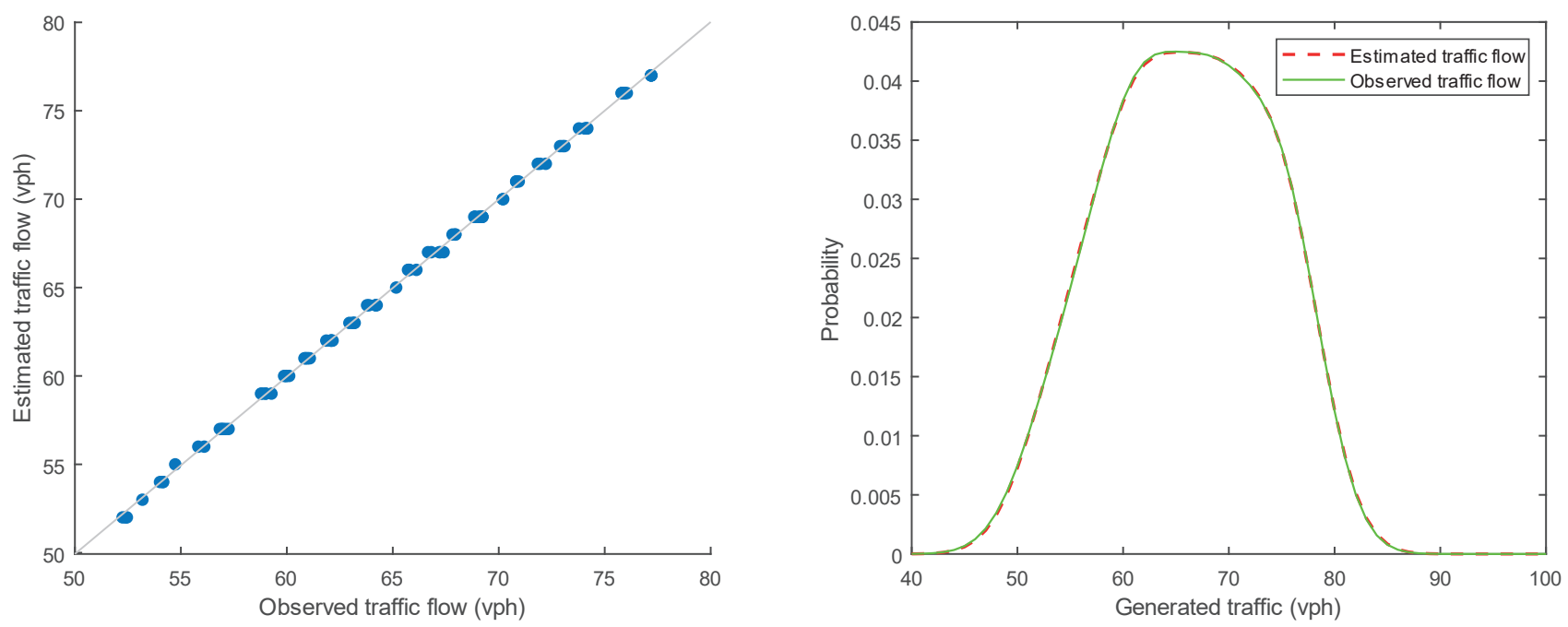

Fig. 8 Illustrating the comparison between the observed traffic flow $\left(q_{2}^{\text {obs. }}\right)$ and estimated traffic flow $\left(q_{2}^{\text {est }}\right)$. The right-side shows probability distributions of them and the left-side shows the cross-validation between them

Table 1 Illustrated the statistical parameters of the $\mathcal{F}_{02}$ set

\begin{tabular}{|c|c|c|c|c|c|c|}
\hline $\begin{array}{l}\text { Link choice set } \\
\left(\mathcal{F}_{02}\right)\end{array}$ & $\begin{array}{c}\text { Base travel } \\
\text { demand }\left(O D^{\text {base }}\right) \\
{[\mathrm{vph}]}\end{array}$ & $\begin{array}{c}\text { Average travel } \\
\text { demand }\left(O D^{\text {ave. }}\right) \\
{[\mathrm{vph}]}\end{array}$ & $\begin{array}{c}\text { Travel demand } \\
\text { variances }\left(O D^{\text {var }}\right) \\
{[\mathrm{vph}]}\end{array}$ & $\begin{array}{c}\operatorname{Bias}(\eta) \\
{[\mathrm{vph}]}\end{array}$ & $\begin{array}{c}\text { Probable travel } \\
\text { demand }\left(O D_{C I=68 \%}^{p o b .}\right) \\
{[\mathrm{vph}]}\end{array}$ & $\begin{array}{c}\text { Probable travel } \\
\text { demand }\left(O D_{C I=95 \%}^{p o b}\right) \\
{[\mathrm{vph}]}\end{array}$ \\
\hline$O D_{04-01}$ & 6.81 & 7.50 & 0.664 & -0.69 & $7.50 \pm 0.815$ & $7.50 \pm 1.597$ \\
\hline$O D_{05-01}$ & 0.49 & 0.54 & 0.003 & -0.05 & $0.54 \pm 0.055$ & $0.54 \pm 0.107$ \\
\hline$O D_{06-01}$ & 6.33 & 6.75 & 0.509 & -0.42 & $6.75 \pm 0.713$ & $6.75 \pm 1.398$ \\
\hline$O D_{07-01}$ & 3.89 & 4.63 & 0.305 & -0.74 & $4.63 \pm 0.552$ & $4.63 \pm 1.082$ \\
\hline$O D_{11-01}$ & 8.76 & 8.69 & 0.755 & +0.07 & $8.69 \pm 0.869$ & $8.69 \pm 1.703$ \\
\hline$O D_{12-01}$ & 5.84 & 7.01 & 0.552 & -1.17 & $7.01 \pm 0.743$ & $7.01 \pm 1.456$ \\
\hline$O D_{16-01}$ & 2.92 & 3.12 & 0.139 & -0.20 & $3.12 \pm 0.373$ & $3.12 \pm 0.731$ \\
\hline$O D_{04-02}$ & 0.74 & 0.80 & 0.008 & -0.06 & $0.80 \pm 0.089$ & $0.80 \pm 0.175$ \\
\hline$O D_{06-02}$ & 0.69 & 0.74 & 0.006 & -0.05 & $0.74 \pm 0.077$ & $0.74 \pm 0.152$ \\
\hline$O D_{07-02}$ & 0.42 & 0.50 & 0.004 & -0.08 & $0.50 \pm 0.063$ & $0.50 \pm 0.124$ \\
\hline$O D_{11-02}$ & 0.95 & 0.96 & 0.010 & -0.01 & $0.96 \pm 0.100$ & $0.96 \pm 0.196$ \\
\hline$O D_{12-02}$ & 0.63 & 0.77 & 0.007 & -0.14 & $0.77 \pm 0.084$ & $0.77 \pm 0.164$ \\
\hline$O D_{16-02}$ & 0.32 & 0.34 & 0.002 & -0.02 & $0.34 \pm 0.045$ & $0.34 \pm 0.088$ \\
\hline$O D_{04-03}$ & 6.45 & 7.33 & 0.617 & -0.88 & $7.33 \pm 0.785$ & $7.33 \pm 1.540$ \\
\hline$O D_{05-03}$ & 0.46 & 0.52 & 0.003 & -0.06 & $0.52 \pm 0.055$ & $0.52 \pm 0.107$ \\
\hline$O D_{11-03}$ & 8.29 & 8.27 & 0.694 & +0.02 & $8.27 \pm 0.833$ & $8.27 \pm 1.633$ \\
\hline$O D_{12-03}$ & 5.53 & 5.97 & 0.403 & -0.44 & $5.97 \pm 0.635$ & $5.97 \pm 1.244$ \\
\hline
\end{tabular}

principally depends on the Inverse-UQ technique based on the least-squares adjustment (LSA) method, that used to calibrate and estimate the distribution parameters (mean and variance) of all elements of the OD matrix. Accordingly, the key contributions of our proposed methodology present: (1) Calibration of the mean values of the OD pairs to reproduce an average-OD matrix. (2) Estimation of the variances of the OD pairs to create a variance-OD matrix. (3) Quantifying the uncertainty of the traffic assignment model using both average-OD and variance-OD matrices.
The methodology was then applied successfully in a case study (Ajka, Hungary). The inputs of the base-OD matrix and the observed traffic flow were used in the Inverse-UQ framework to estimate the posterior distributions of OD pairs. Then, the posterior distributions were employed in Forward-UQ to quantify the traffic flow uncertainty for all links on the transport network. Hence, using this methodology is strongly advised because it enables the decision-makers to expect the variations in traffic flow forecasting. 


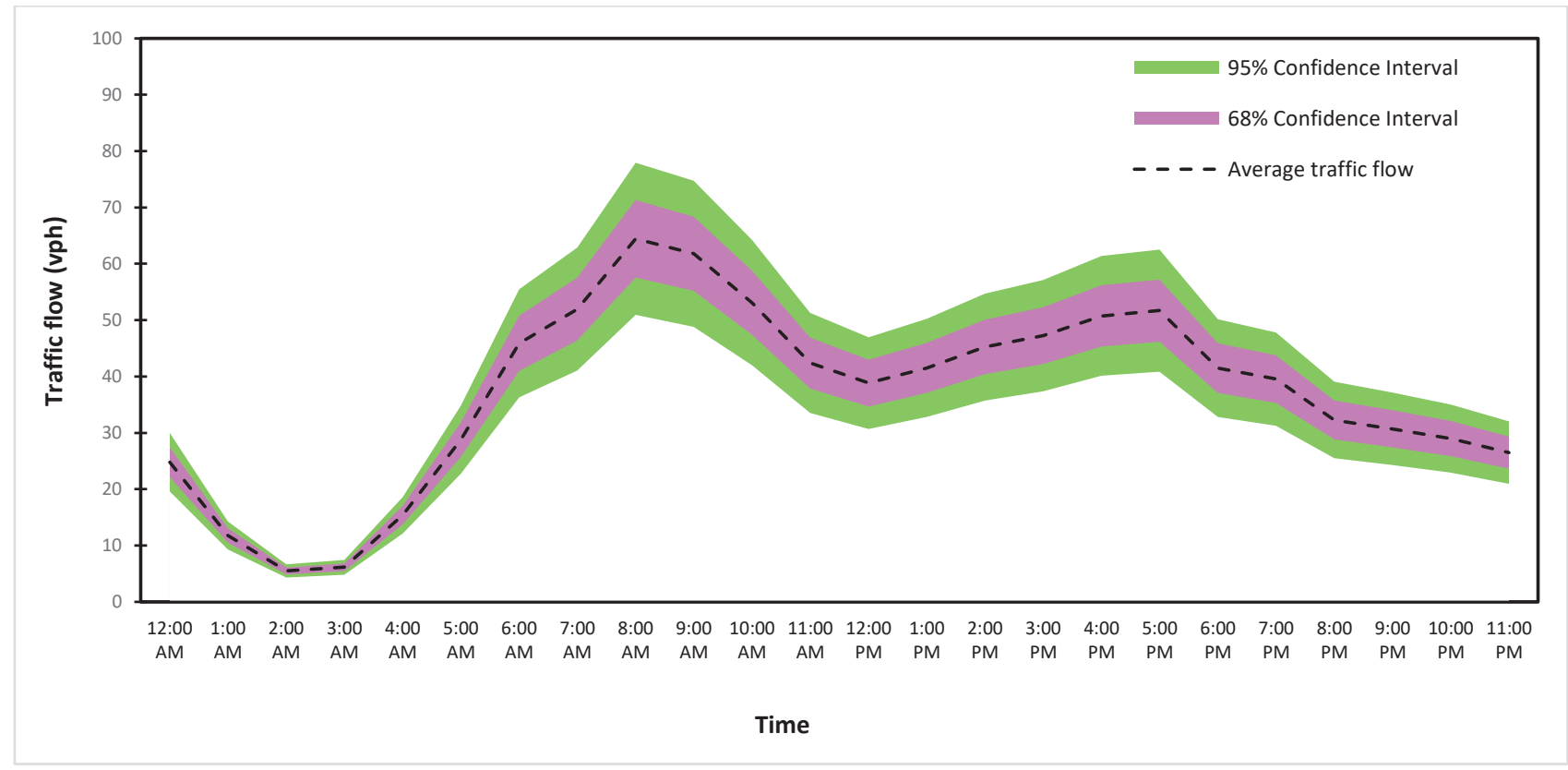

Fig. 9 shows the average traffic flow and the probable traffic flow at $68 \%$ and $95 \%$ confidence interval in link \#2.

Table 2 Illustrated the average traffic flow and the probable traffic flow at $68 \%$ and $95 \%$ confidence interval in link $\# 2$

\begin{tabular}{|c|c|c|c|}
\hline Time & $\begin{array}{c}\text { Average traffic flow }\left(q_{2}^{\text {ave }}\right) \\
{[\mathrm{vph}]}\end{array}$ & $\begin{array}{c}68 \% \mathrm{CI} \\
\text { Probable traffic flow }\left(q_{2}^{\text {prob. }}\right)[\mathrm{vph}]\end{array}$ & $\begin{array}{c}95 \% \mathrm{CI} \\
\text { Probable traffic flow }\left(q_{2}^{p r o b}\right)[\mathrm{vph}]\end{array}$ \\
\hline 12:00-01:00 AM & 24.78 & $24.78 \pm 2.64$ & $24.78 \pm 5.19$ \\
\hline 01:00-02:00 AM & 11.78 & $11.78 \pm 1.36$ & $11.78 \pm 2.57$ \\
\hline 02:00-03:00 AM & 5.51 & $5.51 \pm 0.99$ & $5.51 \pm 1.05$ \\
\hline 03:00-04:00 AM & 6.14 & $6.14 \pm 0.86$ & $6.14 \pm 1.29$ \\
\hline 04:00-05:00 AM & 15.40 & $15.40 \pm 1.65$ & $15.40 \pm 2.63$ \\
\hline 05:00-06:00 AM & 28.77 & $28.77 \pm 3.37$ & $28.77 \pm 6.09$ \\
\hline 06:00-07:00 AM & 45.88 & $45.88 \pm 4.79$ & $45.88 \pm 9.71$ \\
\hline 07:00-08:00 AM & 51.96 & $51.96 \pm 5.51$ & $51.96 \pm 10.98$ \\
\hline 08:00-09:00 AM & 64.44 & $64.44 \pm 6.89$ & $64.44 \pm 13.80$ \\
\hline 09:00-10:00 AM & 61.78 & $61.78 \pm 6.60$ & $61.78 \pm 12.94$ \\
\hline 10:00-11:00 AM & 53.08 & $53.08 \pm 5.67$ & $53.08 \pm 11.02$ \\
\hline 11:00-12:00 AM & 42.39 & $42.39 \pm 4.53$ & $42.39 \pm 8.80$ \\
\hline 12:00-01:00 PM & 38.85 & $38.85 \pm 4.05$ & $38.85 \pm 8.19$ \\
\hline 01:00-02:00 PM & 41.52 & $41.52 \pm 4.54$ & $41.52 \pm 8.55$ \\
\hline 02:00-03:00 PM & 45.23 & $45.23 \pm 4.63$ & $45.23 \pm 9.67$ \\
\hline 03:00-04:00 PM & 47.24 & $47.24 \pm 5.15$ & $47.24 \pm 9.79$ \\
\hline 04:00-05:00 PM & 50.74 & $50.74 \pm 5.26$ & $50.74 \pm 10.83$ \\
\hline 05:00-06:00 PM & 51.69 & $51.69 \pm 5.32$ & $51.69 \pm 11.01$ \\
\hline 06:00-07:00 PM & 41.48 & $41.48 \pm 4.43$ & $41.48 \pm 8.69$ \\
\hline 07:00-08:00 PM & 39.56 & $39.56 \pm 4.35$ & $39.56 \pm 8.05$ \\
\hline 08:00-09:00 AM & 32.28 & $32.28 \pm 3.65$ & $32.28 \pm 6.96$ \\
\hline 09:00-10:00 PM & 30.71 & $30.71 \pm 3.21$ & $30.71 \pm 6.33$ \\
\hline 10:00-11:00 PM & 28.98 & $28.98 \pm 3.16$ & $28.98 \pm 6.17$ \\
\hline 11:00-12:00 PM & 26.49 & $26.49 \pm 2.91$ & $26.49 \pm 5.63$ \\
\hline
\end{tabular}


In this paper, the statistical parameters: mean and variance were handled to characterize the OD pairs uncertainties; this is justified for normal distribution only. However, for other distributions, higher-order statistics parameters

\section{References}

[1] Sullivan, T. J. "Introduction to Uncertainty Quantification", Springer, Cham, Switzerland, 2015.

https://doi.org/10.1007/978-3-319-23395-6

[2] Bigoni, D. "Uncertainty Quantification with Applications to Engineering Problems", PhD Thesis, Technical University of Denmark, 2015.

[3] Litvinenko, A., Matthies, H. G. "Inverse problems and uncertainty quantification", [math.NA], arXiv:1312.5048v2, Cornell University, Ithaca, NY, USA, 2014. [online] Available at: https:/arxiv.org/ abs/1312.5048

[4] Wang, C., Wu, X., Kozlowski, T. "Gaussian Process-Based Inverse Uncertainty Quantification for TRACE Physical Model Parameters Using Steady-State PSBT Benchmark", Nuclear Science and Engineering, 193(1-2), pp. 100-114, 2019. https://doi.org/10.1080/00295639.2018.1499279

[5] Wu, X., Kozlowski, T. "Inverse uncertainty quantification of reactor simulations under the Bayesian framework using surrogate models constructed by polynomial chaos expansion", Nuclear Engineering and Design, 313, pp. 29-52, 2017.

https://doi.org/10.1016/j.nucengdes.2016.11.032

[6] Ghanem, R., Higdon, D., Owhadi, H. "Handbook of Uncertainty Quantification", Springer, Cham, Switzerland, 2017. https://doi.org/10.1007/978-3-319-12385-1

[7] Wu, X., Shirvan, K., Kozlowski, T. "Demonstration of the Relationship between Sensitivity and Identifiability for Inverse Uncertainty Quantification", Journal of Computational Physics, 396, pp. 12-30, 2019. https://doi.org/10.1016/j.jcp.2019.06.032

[8] Cannavó, F. "Sensitivity analysis for volcanic source modeling quality assessment and model selection", Computers and Geosciences, 44, pp. 52-59, 2012. https://doi.org/10.1016/j.cageo.2012.03.008

[9] Nagel, J. B. "Bayesian techniques for inverse uncertainty quantification", PhD Thesis, ETH Zürich, 2017.

https://doi.org/10.3929/ethz-a-010835772

[10] de Jong, G., Daly, A., Pieters, M., Miller, S., Plasmeijer, R., Hofman, F. "Uncertainty in traffic forecasts: literature review and new results for The Netherlands", Transportation, 34, pp. 375-395, 2007. https://doi.org/10.1007/s11116-006-9110-8

[11] Ashley, D. J. "Uncertainty in the context of highway appraisal", Transportation, 9, pp. 249-267, 1980. https://doi.org/10.1007/bf00153867

[12] Lowe, S. R., Morrell, D., Copley, G. "Uncertainty in Highway Appraisal: the Development of Systematic Sensitivity Testing", In: Highway Planning and Design, Proceedings of the Seminar K, held at the PTRC's 10th Annual Summer Meeting, Warwick University, Coventry, UK, 1982, pp. 15-29. are needed to improve the characterization. How to make use of higher-order statistics to enhance the quality of UQ will be a significant future research direction.

[13] Kroes, E. P. "Some Issues in Traffic Forecasting for Privately Financed Infrastructure", presented at Pan-European Transport Issues: Proceedings of Seminar A Held at the PTRC European Transport Forum, Brunel University, London, UK, Sept. 2-6, 1996.

[14] Leurent, F. "An analysis of modelling error, with application to a traffic assignment model with continuously distributed values of time", presented at Transportation Planning Methods: Proceedings of Seminar D \& E Held at the PTRC European Transport Forum, Brunel University, London, UK, Sept. 2-6, 1996.

[15] Leurent, F. "Sensitivity and error analysis of the dual criteria traffic assignment model", Transportation Research Part B: Methodological, 32(3), pp. 189-204, 1998. https://doi.org/10.1016/s0191-2615(97)00024-6

[16] de Jong, G. C., Ettema, D. F., Gunn, H. F., Cheung, Y. H. F., Kleijn, H. J. "Preparatory research into updating values of travel time in The Netherlands", presented at 8th WCTR Conference, Antwerp, The Netherlands, July, 12-17, 1998.

[17] Zhang, H., Pu, Z. "Beating the Uncertainties: Ensemble Forecasting and Ensemble-Based Data Assimilation in Modern Numerical Weather Prediction", Advances in Meteorology, 2010, Article ID 432160, 2010. https://doi.org/10.1155/2010/432160

[18] Perrakis, K., Cools, M., Karlis, D., Janssens, D., Kochan, B., Bellemans, T., Wets, G. "Quantifying Input Uncertainty in Traffic Assignment Models", presented at Proceedings of the 91st Annual Meeting of the Transportation Research Board, Washingon, DC, USA, Jan. 22-26, 2012.

[19] Deng, W., Lei, H., Zhou, X. "Traffic state estimation and uncertainty quantification based on heterogeneous data sources: A three detector approach", Transportation Research Part B: Methodological, 57, pp. 132-157, 2013.

https://doi.org/10.1016/j.trb.2013.08.015

[20] Soetaert, K., Petzoldt, T. "Inverse Modelling, Sensitivity and Monte Carlo Analysis in R Using Package FME", Journal of Statistical Software, 33(3), 2010. https://doi.org/10.18637/jss.v033.i03

[21] Frey, H. C., Rhodes, D. S. "Quantitative Analysis of Variability and Uncertainty in Environmental Data and Models. Volume 1. Theory and Methodology Based Upon Bootstrap Simulation", North Carolina State University, Raleigh, NC, USA, Rep. DOE/ER/30250Vol.1, 1999. https://doi.org/10.2172/1178941

[22] Smith, R. C. "Uncertainty quantification: theory, implementation, and applications", Society for Industrial and Applied Mathematics, Philadelphia, PA, USA, 2013.

[23] Zio, E., Pedroni, N. "Methods for representing uncertainty", Foundation for an Industrial Safety Culture, Toulouse, France, 2013. [online] Available at: https://www.foncsi.org/fr/publications/ cahiers-securite-industrielle/literature-review-uncertainty-representation/CSI-uncertainty-representation.pdf 
[24] Hammonds, J. S., Hoffman, F. O., Bartell, S. M. "An introductory guide to uncertainty analysis in environmental and health risk assessment. Environmental Restoration Program", Senes Oak Ridge, Inc., Oak Ridge, TN, USA, Rep. ES/ER/TM-35/R1, 1994. https://doi.org/10.2172/10127301

[25] Lin, G., Engel, D. W., Eslinger, P. W. "Survey and Evaluate Uncertainty Quantification Methodologies", Pacific Northwest National Laboratory (PNNL), Richland, WA, USA, Rep. PNNL-20914, 2012. https://doi.org/10.2172/1035732

[26] Yoo, E.-H. E., Aldstadt, J. "Principles of Modeling Uncertainties in Spatial Data and Spatial Analyses by Wenzhong Shi", Journal of Regional Science, 51(4), pp. 860-861, 2011. https://doi.org/10.1111/j.1467-9787.2011.00740_14.x

[27] Hand, D. J. "Measurements and their Uncertainties: A Practical Guide to Modern Error Analysis by Ifan G. Hughes, Thomas P. A. Hase", International Statistical Review, 79(2), pp. 280-280, 2011. https://doi.org/10.1111/j.1751-5823.2011.00149_8.x

[28] Wu, X., Kozlowski, T., Meidani, H., Shirvan, K. "Inverse uncertainty quantification using the modular Bayesian approach based on Gaussian process, Part 1: Theory", Nuclear Engineering and Design, 335, pp. 339-355, 2018.

https://doi.org/10.1016/j.nucengdes.2018.06.004
[29] Iglesias, M. A., Lin, K., Stuart, A. M. "Well-posed Bayesian geometric inverse problems arising in subsurface flow", Inverse Problems, 30(11), Article number: 114001, 2014. https://doi.org/10.1088/0266-5611/30/11/114001

[30] Seeger, M. "Gaussian Processes for Machine Learning", International Journal of Neural Systems, 14(2), pp. 69-106, 2004. https://doi.org/10.1142/s0129065704001899

[31] Navratil, G. "Adjustment Computations: Spatial Data Analysis", International Journal of Geographical Information Science, 25(2), pp. 26-327, 2011. https://doi.org/10.1080/13658816.2010.501335

[32] Dermanis, A., Grün, A., Sansó, F. "Geomatic Method for the Analysis of Data in the Earth Sciences", Springer, Berlin, Heidelberg, Germany, 2000. https://doi.org/10.1007/3-540-45597-3

[33] Wolberg, J. "Data Analysis Using the Method of Least Squares", Springer, Berlin, Heidelberg, Germany, 2006. https://doi.org/10.1007/3-540-31720-1

[34] Ogundare, J. O. "Understanding Least Squares Estimation and Geomatics Data Analysis", John Wiley \& Sons, Hoboken, NJ, USA, 2018.

https://doi.org/10.1002/9781119501459 


\section{Appendix A}

\begin{tabular}{l}
\hline Algorithm 1 Inverse-UQ proce \\
\hline Data stage: \\
$Z_{i=1, n} ; \quad \forall Z, \quad i \in \mathbb{N}$ \\
$O_{i=1, n} ; \quad O \subset Z$ \\
$D_{j=1, n} ; \quad D \subset Z$ \\
$P=Z^{2} ; \quad P \in \mathbb{N}$ \\
$O D_{p=1, P}^{o b s .} ; \quad \forall O D_{p=1, P}^{o b s .} \in \mathbb{R}$ \\
$q_{l=1, L}^{o b s .} ; \quad \forall q_{l=1, L}^{o b s .} \in \mathbb{R}$ \\
$L ; L \in \mathbb{N}$ \\
$\mathcal{F}_{l=1, L} ;$ \\
$\beta_{l=1, L} ;$ \\
$f_{T O \rightarrow D}$ \\
$N ; N \in \mathbb{N}$ \\
Min.RSS
\end{tabular}

\section{Calibration process stage:}

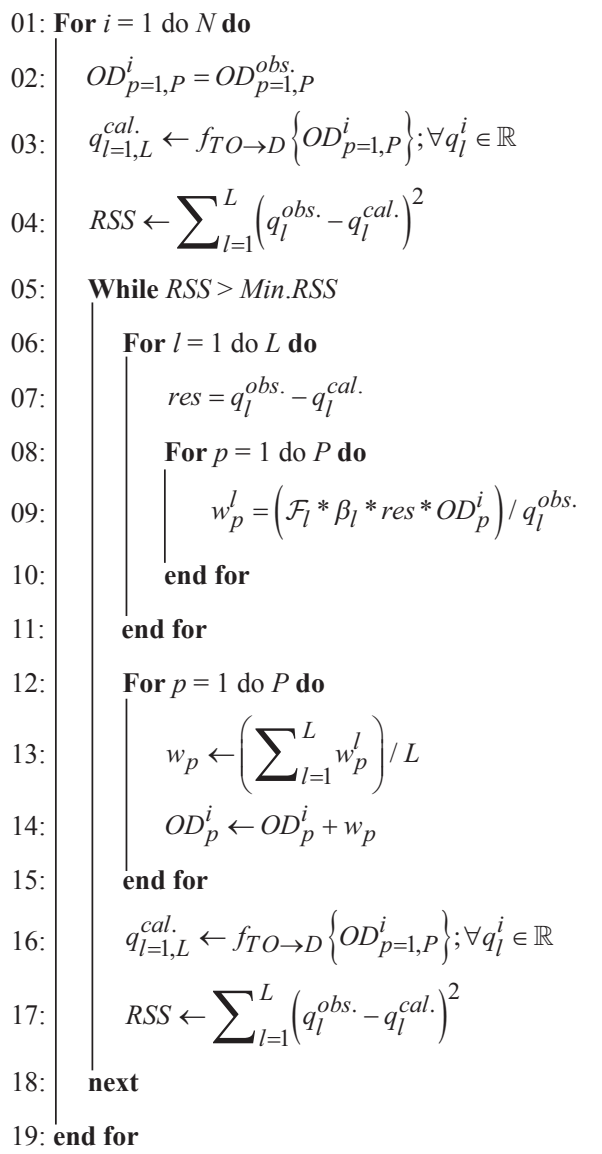
$\triangleright$ Zone definition.
$\triangleright$ Origin definition.
$\triangleright$ Destination definition.
$\triangleright$ Number of OD pairs.
$\triangleright$ Observed values of OD matrix.
$\triangleright$ Observed traffic flow.
$\triangleright$ Number of links.
$\triangleright$ Link choice set.
$\triangleright$ Link choice probabilites set.
$\triangleright$ Traffic assignment model.
$\triangleright$ Samples size.
$\triangleright$ Minimum residual sum of squares.

$\triangleright$ Run VISUM.

$\triangleright$ Find the residual sum of squares.

$\triangleright$ Find the residual.

$\triangleright$ Find the weight of the OD pairs for each link.

$\triangleright$ Find the calibrated OD pairs.

$\triangleright$ Run VISUM.

$\triangleright$ Find the residual sum of squares.

\section{Results stage:}
20: $O D_{p=1, P}^{\text {ave }} \leftarrow\left(\sum_{i=1}^{N} O D_{p=1, P}^{i} O D_{p=1, P}^{i}\right) / N$
$\triangleright$ Find the average OD matrix.
21: $O D_{p=1, P}^{\text {var. }} \leftarrow\left(\sum_{i=1}^{N}\left(O D_{p=1, P}^{i}-O D_{p=1, P}^{a v e .}\right)^{2}\right) / N$
$\triangleright$ Find the variance OD matrix. 


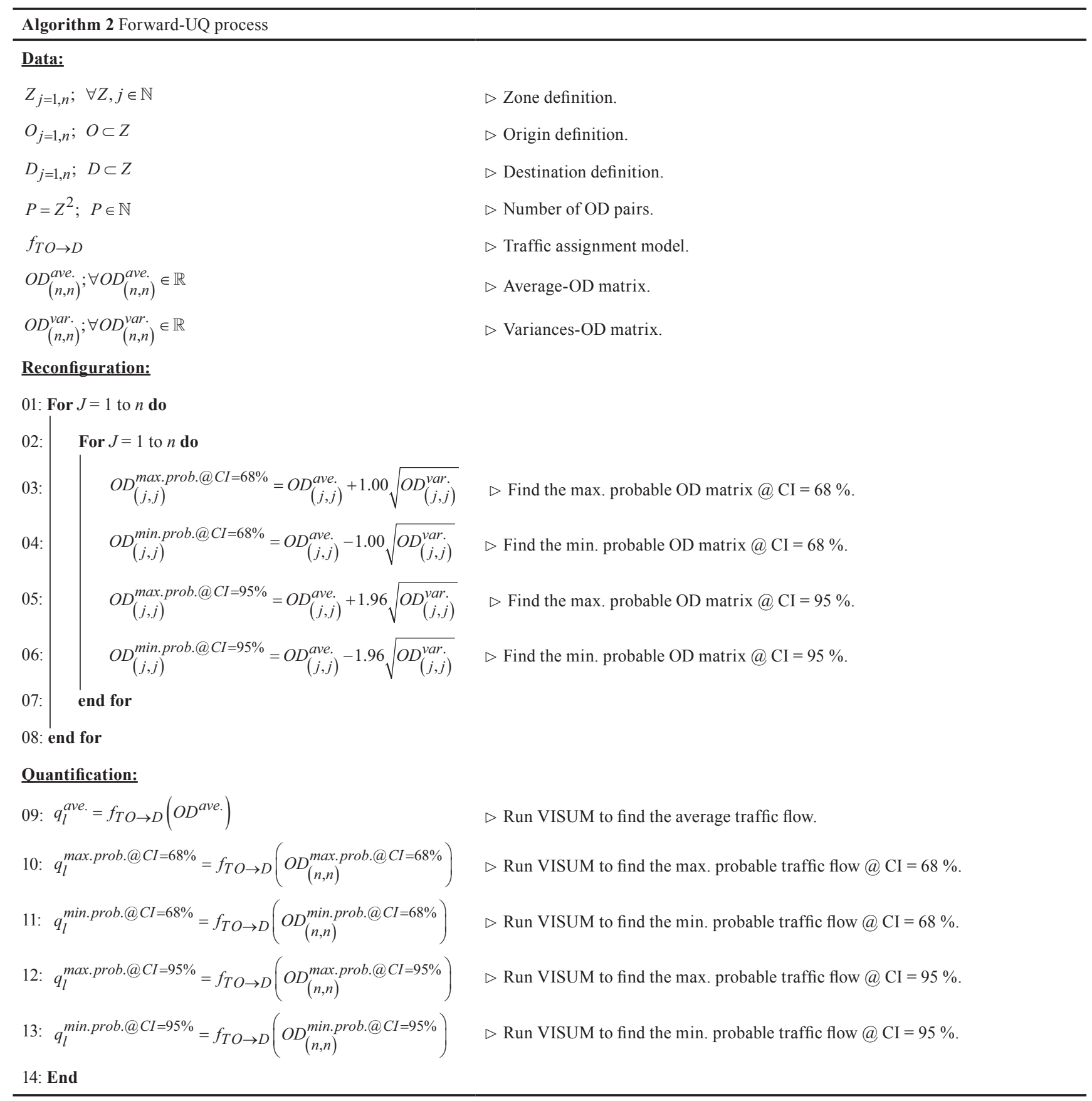




\section{Appendix B}

Table 3 The base-OD matrix

\begin{tabular}{|c|c|c|c|c|c|c|c|c|c|c|c|c|c|c|c|c|c|c|c|c|c|c|c|c|c|}
\hline & Z 01 & Z 02 & Z 03 & Z 04 & Z 05 & Z 06 & Z 07 & Z 08 & Z 09 & Z 10 & Z 11 & Z 12 & Z 13 & Z 14 & Z 15 & Z 16 & Z 17 & Z 18 & Z 19 & Z 20 & Z 21 & Z 22 & Z 23 & Z 24 & Z 25 \\
\hline-01 & 0.0 & 30.1 & 263.2 & 7.2 & 0.6 & 6.7 & 4.1 & 5.6 & 8.2 & 6.1 & 9.2 & 6.1 & 5.6 & 5.6 & 3.6 & 3.1 & 3.6 & 3.6 & 6.1 & 6.1 & 3.6 & 3.1 & 13.8 & 9.7 & 7.2 \\
\hline Z-02 & 9.1 & 0.0 & 8.6 & 0.2 & 0.0 & 0.2 & 0.1 & 0.2 & 0.3 & 0.2 & 0.3 & 0.2 & 0.2 & 0.2 & 0.1 & 0.1 & 0.1 & 0.1 & 0.2 & 0.2 & 0.1 & 0.1 & 0.5 & 0.3 & 0.2 \\
\hline Z-03 & 268.6 & 29.1 & 0.0 & 6.9 & 0.6 & 6.4 & 4.0 & 5.4 & 7.9 & 5.9 & 8.9 & 5.9 & 5.4 & 5.4 & 3.5 & 3.0 & 3.5 & 3.5 & 5.9 & 5.9 & 3.5 & 3.0 & 13.4 & 9.4 & 6.9 \\
\hline Z-04 & 6.8 & 0.7 & 6.4 & 0.0 & 0.0 & 0.0 & 0.0 & 0.0 & 0.0 & 0.0 & 0.0 & 0.0 & 0.0 & 0.0 & 0.0 & 0.0 & 0.0 & 0.0 & 0.0 & 0.0 & 0.0 & 0.0 & 0.0 & 0.0 & 0.0 \\
\hline Z-05 & 0.5 & 0.1 & 0.5 & 0.0 & 0.0 & 0.0 & 0.0 & 0.0 & 0.0 & 0.0 & 0.0 & 0.0 & 0.0 & 0.0 & 0.0 & 0.0 & 0.0 & 0.0 & 0.0 & 0.0 & 0.0 & 0.0 & 0.0 & 0.0 & 0.0 \\
\hline Z-06 & 6.3 & 0.7 & 6.0 & 0.0 & 0.0 & 0.0 & 0.0 & 0.0 & 0.0 & 0.0 & 0.0 & 0.0 & 0.0 & 0.0 & 0.0 & 0.0 & 0.0 & 0.0 & 0.0 & 0.0 & 0.0 & 0.0 & 0.0 & 0.0 & 0.0 \\
\hline Z-07 & 3.9 & 0.4 & 3.7 & 0.0 & 0.0 & 0.0 & 0.0 & 0.0 & 0.0 & 0.0 & 0.0 & 0.0 & 0.0 & 0.0 & 0.0 & 0.0 & 0.0 & 0.0 & 0.0 & 0.0 & 0.0 & 0.0 & 0.0 & 0.0 & 0.0 \\
\hline Z-08 & 5.4 & 0.6 & 5.1 & 0.0 & 0.0 & 0.0 & 0.0 & 0.0 & 0.0 & 0.0 & 0.0 & 0.0 & 0.0 & 0.0 & 0.0 & 0.0 & 0.0 & 0.0 & 0.0 & 0.0 & 0.0 & 0.0 & 0.0 & 0.0 & 0.0 \\
\hline Z-09 & 7.8 & 0.8 & 7.4 & 0.0 & 0.0 & 0.0 & 0.0 & 0.0 & 0.0 & 0.0 & 0.0 & 0.0 & 0.0 & 0.0 & 0.0 & 0.0 & 0.0 & 0.0 & 0.0 & 0.0 & 0.0 & 0.0 & 0.0 & 0.0 & 0.0 \\
\hline Z-10 & 5.8 & 0.6 & 5.5 & 0.0 & 0.0 & 0.0 & 0.0 & 0.0 & 0.0 & 0.0 & 0.0 & 0.0 & 0.0 & 0.0 & 0.0 & 0.0 & 0.0 & 0.0 & 0.0 & 0.0 & 0.0 & 0.0 & 0.0 & 0.0 & 0.0 \\
\hline Z-11 & 8.8 & 0.9 & 8.3 & 0.0 & 0.0 & 0.0 & 0.0 & 0.0 & 0.0 & 0.0 & 0.0 & 0.0 & 0.0 & 0.0 & 0.0 & 0.0 & 0.0 & 0.0 & 0.0 & 0.0 & 0.0 & 0.0 & 0.0 & 0.0 & 0.0 \\
\hline Z-12 & 5.8 & 0.6 & 5.5 & 0.0 & 0.0 & 0.0 & 0.0 & 0.0 & 0.0 & 0.0 & 0.0 & 0.0 & 0.0 & 0.0 & 0.0 & 0.0 & 0.0 & 0.0 & 0.0 & 0.0 & 0.0 & 0.0 & 0.0 & 0.0 & 0.0 \\
\hline Z-13 & 5.4 & 0.6 & 5.1 & 0.0 & 0.0 & 0.0 & 0.0 & 0.0 & 0.0 & 0.0 & 0.0 & 0.0 & 0.0 & 0.0 & 0.0 & 0.0 & 0.0 & 0.0 & 0.0 & 0.0 & 0.0 & 0.0 & 0.0 & 0.0 & 0.0 \\
\hline Z-14 & 5.4 & 0.6 & 5.1 & 0.0 & 0.0 & 0.0 & 0.0 & 0.0 & 0.0 & 0.0 & 0.0 & 0.0 & 0.0 & 0.0 & 0.0 & 0.0 & 0.0 & 0.0 & 0.0 & 0.0 & 0.0 & 0.0 & 0.0 & 0.0 & 0.0 \\
\hline Z-15 & 3.4 & 0.4 & 3.2 & 0.0 & 0.0 & 0.0 & 0.0 & 0.0 & 0.0 & 0.0 & 0.0 & 0.0 & 0.0 & 0.0 & 0.0 & 0.0 & 0.0 & 0.0 & 0.0 & 0.0 & 0.0 & 0.0 & 0.0 & 0.0 & 0.0 \\
\hline Z-16 & 2.9 & 0.3 & 2.8 & 0.0 & 0.0 & 0.0 & 0.0 & 0.0 & 0.0 & 0.0 & 0.0 & 0.0 & 0.0 & 0.0 & 0.0 & 0.0 & 0.0 & 0.0 & 0.0 & 0.0 & 0.0 & 0.0 & 0.0 & 0.0 & 0.0 \\
\hline Z-17 & 3.4 & 0.4 & 3.2 & 0.0 & 0.0 & 0.0 & 0.0 & 0.0 & 0.0 & 0.0 & 0.0 & 0.0 & 0.0 & 0.0 & 0.0 & 0.0 & 0.0 & 0.0 & 0.0 & 0.0 & 0.0 & 0.0 & 0.0 & 0.0 & 0.0 \\
\hline Z-18 & 3.4 & 0.4 & 3.2 & 0.0 & 0.0 & 0.0 & 0.0 & 0.0 & 0.0 & 0.0 & 0.0 & 0.0 & 0.0 & 0.0 & 0.0 & 0.0 & 0.0 & 0.0 & 0.0 & 0.0 & 0.0 & 0.0 & 0.0 & 0.0 & 0.0 \\
\hline Z-19 & 5.8 & 0.6 & 5.5 & 0.0 & 0.0 & 0.0 & 0.0 & 0.0 & 0.0 & 0.0 & 0.0 & 0.0 & 0.0 & 0.0 & 0.0 & 0.0 & 0.0 & 0.0 & 0.0 & 0.0 & 0.0 & 0.0 & 0.0 & 0.0 & 0.0 \\
\hline Z-20 & 5.8 & 0.6 & 5.5 & 0.0 & 0.0 & 0.0 & 0.0 & 0.0 & 0.0 & 0.0 & 0.0 & 0.0 & 0.0 & 0.0 & 0.0 & 0.0 & 0.0 & 0.0 & 0.0 & 0.0 & 0.0 & 0.0 & 0.0 & 0.0 & 0.0 \\
\hline Z-21 & 3.4 & 0.4 & 3.2 & 0.0 & 0.0 & 0.0 & 0.0 & 0.0 & 0.0 & 0.0 & 0.0 & 0.0 & 0.0 & 0.0 & 0.0 & 0.0 & 0.0 & 0.0 & 0.0 & 0.0 & 0.0 & 0.0 & 0.0 & 0.0 & 0.0 \\
\hline Z-22 & 2.9 & 0.3 & 2.8 & 0.0 & 0.0 & 0.0 & 0.0 & 0.0 & 0.0 & 0.0 & 0.0 & 0.0 & 0.0 & 0.0 & 0.0 & 0.0 & 0.0 & 0.0 & 0.0 & 0.0 & 0.0 & 0.0 & 0.0 & 0.0 & 0.0 \\
\hline Z-23 & 13.1 & 1.4 & 12.4 & 0.0 & 0.0 & 0.0 & 0.0 & 0.0 & 0.0 & 0.0 & 0.0 & 0.0 & 0.0 & 0.0 & 0.0 & 0.0 & 0.0 & 0.0 & 0.0 & 0.0 & 0.0 & 0.0 & 0.0 & 0.0 & 0.0 \\
\hline Z-24 & 9.2 & 1.0 & 8.8 & 0.0 & 0.0 & 0.0 & 0.0 & 0.0 & 0.0 & 0.0 & 0.0 & 0.0 & 0.0 & 0.0 & 0.0 & 0.0 & 0.0 & 0.0 & 0.0 & 0.0 & 0.0 & 0.0 & 0.0 & 0.0 & 0.0 \\
\hline Z-25 & 6.8 & 0.7 & 6.4 & 0.0 & 0.0 & 0.0 & 0.0 & 0.0 & 0.0 & 0.0 & 0.0 & 0.0 & 0.0 & 0.0 & 0.0 & 0.0 & 0.0 & 0.0 & 0.0 & 0.0 & 0.0 & 0.0 & 0.0 & 0.0 & 0.0 \\
\hline
\end{tabular}


Table 4 The average-OD matrix

\begin{tabular}{|c|c|c|c|c|c|c|c|c|c|c|c|c|c|c|c|c|c|c|c|c|c|c|c|c|c|}
\hline$V$ & Z 01 & Z 02 & Z 03 & Z 04 & Z 05 & Z 06 & Z 07 & Z 08 & Z 09 & Z 10 & Z 11 & Z 12 & Z 13 & Z 14 & Z 15 & Z 16 & Z 17 & Z 18 & Z 19 & Z 20 & Z 21 & Z 22 & Z 23 & Z 24 & Z 25 \\
\hline 01 & 0.0 & 31.6 & 292.6 & 3 & 0. & 7.6 & 4 & 5.6 & 3 & 7.2 & 8 & 7.4 & 2 & 6.9 & .9 & 3.5 & 3.3 & 3.3 & 11.9 & 5.8 & 7.2 & 3.4 & 14.5 & 11.5 & 7.2 \\
\hline Z-02 & 11.8 & 0.0 & 9.6 & 0.3 & 0.0 & 0.2 & \begin{tabular}{|l|}
0.1 \\
\end{tabular} & 0.2 & 0.3 & 0.2 & 0.3 & 0.2 & 0.2 & 0.2 & 0.1 & 0.1 & 0.1 & 0.1 & 0.4 & 0.2 & 0.2 & 0.1 & 0.5 & 0.5 & 0.3 \\
\hline Z-03 & 301.1 & 32.7 & 0.0 & 8.6 & 0.7 & 7.0 & 4.1 & 6.3 & 7.8 & 6.2 & 9.7 & 6.7 & 6.1 & 5.9 & 3.9 & 3.9 & 3.5 & 3.9 & 7.0 & 6.7 & 0.8 & 3.0 & 15.0 & 10.5 & 7.8 \\
\hline-04 & 7.5 & 0.8 & 7.3 & 0.0 & \begin{tabular}{|l|l} 
& 0.0
\end{tabular} & 0.0 & \begin{tabular}{|l|}
0.0 \\
\end{tabular} & 0.0 & 0.0 & 0.0 & \begin{tabular}{|l|l} 
& 0.0
\end{tabular} & 0.0 & 0.0 & 0.0 & 0.0 & 0.0 & 0.0 & 0.0 & 0.0 & 0.0 & 0.0 & 0.0 & 0.0 & 0.0 & 0.0 \\
\hline-05 & 0.5 & 0.1 & 0.5 & 0.0 & 0.0 & 0.0 & 0.0 & 0.0 & 0.0 & 0.0 & 0.0 & 0.0 & 0.0 & 0.0 & 0.0 & 0.0 & 0.0 & 0.0 & 0.0 & 0.0 & 0.0 & 0.0 & 0.0 & 0.0 & 0.0 \\
\hline 06 & 6.8 & 0.7 & 7.0 & 0.0 & 0.0 & 0.0 & 0.0 & 0.0 & 0.0 & 0.0 & 0.0 & 0.0 & 0.0 & 0.0 & 0.0 & 0.0 & 0.0 & 0.0 & 0.0 & 0.0 & 0.0 & 0.0 & 0.0 & 0.0 & 0.0 \\
\hline 07 & 4.6 & 0.5 & 4.7 & 0.0 & \begin{tabular}{|l|}
0.0 \\
\end{tabular} & 0.0 & 0.0 & 0.0 & 0.0 & 0.0 & 0.0 & 0.0 & 0.0 & 0.0 & 0.0 & 0.0 & 0.0 & 0.0 & 0.0 & 0.0 & 0.0 & 0.0 & 0.0 & 0.0 & 0.0 \\
\hline 08 & 5.8 & 0.6 & 5.0 & 0.0 & 0.0 & 0.0 & 0.0 & 0.0 & 0.0 & 0.0 & .0 & 0.0 & 0.0 & 0.0 & 0.0 & 0.0 & 0.0 & 0.0 & 0.0 & 0.0 & 0.0 & .0 & 0.0 & .0 & 0.0 \\
\hline 09 & 8.5 & 0.9 & 8.1 & 0.0 & 0.0 & 0.0 & 0. & 0.0 & 0.0 & 0.0 & 0.0 & 0.0 & 0.0 & 0. & 0.0 & 0.0 & 0.0 & 0.0 & 0.0 & 0.0 & 0.0 & .0 & 0.0 & .0 & .0 \\
\hline Z-10 & 6.7 & 0.7 & 6.2 & 0.0 & 0.0 & 0.0 & 0.0 & 0.0 & 0.0 & 0.0 & 0.0 & 0.0 & 0.0 & 0.0 & 0.0 & 0.0 & 0.0 & 0.0 & 0.0 & 0.0 & 0.0 & 0.0 & 0.0 & 0.0 & 0.0 \\
\hline Z-11 & 8.7 & 1.0 & 8.3 & 0.0 & 0.0 & 0.0 & 0.0 & 0.0 & 0.0 & 0.0 & 0.0 & 0.0 & 0.0 & 0.0 & 0.0 & 0.0 & 0.0 & 0.0 & 0.0 & 0.0 & 0.0 & 0.0 & 0.0 & 0.0 & 0.0 \\
\hline 12 & 7.0 & 0.8 & 6.0 & 0.0 & 0.0 & 0.0 & 0.0 & 0.0 & 0.0 & 0.0 & 0.0 & 0.0 & 0.0 & 0.0 & 0 & 0.0 & 0.0 & 0.0 & 0.0 & 0.0 & 0.0 & 0.0 & 0.0 & .0 & 0.0 \\
\hline Z-13 & 5.9 & 0.6 & 50 & 0.0 & 0.0 & 0.0 & 0 & 0.0 & 0 & 0.0 & 0 & 0.0 & 0.0 & 0.0 & 0 & 0.0 & 0.0 & 0.0 & 0.0 & 0.0 & 0.0 & 0.0 & 0.0 & 0.0 & 0 \\
\hline-14 & 6.0 & 0.6 & 5.6 & 0.0 & 0.0 & 0.0 & 0.0 & 0.0 & 0.0 & 0.0 & 0.0 & 0.0 & 0.0 & 0.0 & 0.0 & 0.0 & 0.0 & 0.0 & 0.0 & 0.0 & 0.0 & 0.0 & 0.0 & 0.0 & 0.0 \\
\hline-15 & 3.8 & 0.4 & 3.7 & 0.0 & 0.0 & 0.0 & 0.0 & 0.0 & 0.0 & 0.0 & 0.0 & 0.0 & 0.0 & 0.0 & 0.0 & 0.0 & 0.0 & 0.0 & 0.0 & 0.0 & 0.0 & 0.0 & 0.0 & 0.0 & 0.0 \\
\hline 16 & 3.1 & 0.3 & 2.8 & \begin{tabular}{|l|l|} 
& 0.0
\end{tabular} & \begin{tabular}{|l|l|} 
& 0.0
\end{tabular} & \begin{tabular}{|l|l|} 
& 0.0
\end{tabular} & $\mid$\begin{tabular}{|l|l} 
\\
\end{tabular} & 0.0 & 0.0 & 0.0 & 0.0 & 0.0 & 0.0 & 0.0 & 0.0 & 0.0 & 0.0 & 0.0 & 0.0 & 0.0 & 0.0 & 0.0 & 0.0 & 0.0 & 0.0 \\
\hline Z-17 & 3.5 & 1.5 & 3.6 & 0.0 & 0.0 & 0.0 & 0.0 & 0.0 & 0.0 & 0.0 & 0.0 & 0.0 & 0 & 0.0 & 0 & 0.0 & 0.0 & 0.0 & 0.0 & 0 & 0.0 & 0.0 & 0.0 & 0.0 & 0.0 \\
\hline 18 & 3.3 & 0.4 & 3. & 0.0 & 0. & 0.0 & 0. & 0.0 & 0.0 & 0.0 & 0.0 & 0.0 & 0.0 & 0.0 & 8 & 0.0 & 0.0 & 0.0 & 0.0 & 8 & 0.0 & .0 & 0.0 & 0.0 & 0.0 \\
\hline Z-19 & 3.1 & 0.2 & 4.9 & 0.0 & 0.0 & 0.0 & 0.0 & 0.0 & 0.0 & 0.0 & 0.0 & 0.0 & 0.0 & 0.0 & 0.0 & 0.0 & 0.0 & 0.0 & 0.0 & 0.0 & 0.0 & 0.0 & 0.0 & 0.0 & 0.0 \\
\hline 20 & 5.8 & 0.6 & 6.2 & \begin{tabular}{|l|l} 
\\
\end{tabular} & \begin{tabular}{|l|l} 
\\
\end{tabular} & \begin{tabular}{|l|l} 
& 0.0 \\
\end{tabular} & \begin{tabular}{|l|l} 
& 0.0 \\
\end{tabular} & 0.0 & 0.0 & 0.0 & 0.0 & 0.0 & 0.0 & 0.0 & 0.0 & 0.0 & 0.0 & 0.0 & 0.0 & 0.0 & 0.0 & 0.0 & 0.0 & 0.0 & 0.0 \\
\hline Z-21 & 5.3 & 0. & 0.6 & 0. & 0. & 0. & 0.0 & 0. & 0.0 & 0. & 0.0 & 0. & 0.0 & 0. & 0.0 & 0.0 & 0.0 & 0.0 & 0.0 & 0.0 & 0.0 & 0 & 0.0 & .0 & .0 \\
\hline Z-22 & 3.3 & 0.4 & 2.8 & 0.0 & \begin{tabular}{|l|l} 
\\
\end{tabular} & 0.0 & 0.0 & 0.0 & 0.0 & 0.0 & 0.0 & 0.0 & 0.0 & 0.0 & 0.0 & 0.0 & 0.0 & 0.0 & 0.0 & 0.0 & 0.0 & 0.0 & 0.0 & 0.0 & 0.0 \\
\hline Z-23 & 17.0 & 1.4 & 13.8 & 0.0 & 0.0 & 0.0 & 0.0 & 0.0 & 0.0 & 0.0 & 0.0 & 0.0 & 0.0 & 0.0 & 0.0 & 0.0 & 0.0 & 0.0 & 0.0 & 0.0 & 0.0 & 0.0 & 0.0 & 0.0 & 0.0 \\
\hline Z-24 & 9.5 & 1.0 & 9.7 & \begin{tabular}{|l|l}
0.0 \\
\end{tabular} & \begin{tabular}{|l|l}
0.0 \\
\end{tabular} & \begin{tabular}{|l|l}
0.0 \\
\end{tabular} & 0.0 & 0.0 & 0.0 & 0.0 & 0.0 & 0.0 & 0.0 & 0.0 & 0.0 & 0.0 & 0.0 & 0.0 & 0.0 & 0.0 & 0.0 & 0.0 & 0.0 & 0.0 & 0.0 \\
\hline Z-25 & 6.8 & 0.8 & 7.2 & 0.0 & 0.0 & 0.0 & 0.0 & 0.0 & 0.0 & 0.0 & 0.0 & 0.0 & 0.0 & 0.0 & 0.0 & 0.0 & 0.0 & 0.0 & 0.0 & 0.0 & 0.0 & 0.0 & 0.0 & 0.0 & 0.0 \\
\hline
\end{tabular}


Table 5 The variance-OD matrix

\begin{tabular}{|c|c|c|c|c|c|c|c|c|c|c|c|c|c|c|c|c|c|c|c|c|c|c|c|c|c|}
\hline & Z 01 & 2 & 3 & 04 & 05 & 06 & 07 & 08 & Z 09 & Z 10 & Z 11 & Z 12 & Z 13 & Z 14 & Z 15 & Z 16 & Z 17 & 18 & 19 & 20 & 21 & Z 22 & 23 & Z 24 & Z 25 \\
\hline 01 & & & & & & & & & & & & & & & & & & & & & & & & & \\
\hline 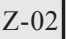 & 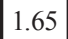 & & 06 & 00 & & & & & 0 & & & & & & & & & 0 & 0 & 0 & 00 & 00 & 0 & .00 & .00 \\
\hline 93 & 35 & 31 & 50 & 86 & & & & & 0.73 & & 7 & 51 & & & & 23 & & & 55 & 51 & 14 & 00 & .60 & .26 & 69 \\
\hline 04 & 66 & 01 & 62 & 00 & 00 & & & & 00 & & 0 & 00 & 0 & 0 & & 00 & 0 & 0 & 00 & 00 & 00 & 00 & 0.00 & 0.00 & .00 \\
\hline 05 & 00 & 00 & 50 & 00 & 50 & 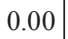 & ( & 00 & 00 & 00 & 00 & 00 & .00 & 0.00 & 00 & 00 & ( & .00 & 00 & 00 & 00 & 0.00 & .00 & .00 & O \\
\hline Z-06 & 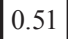 & & 71 & 00 & 50 & 0 & 0 & 00 & 00 & 00 & 00 & 0 & 0 & J & 50 & 0 & 0.00 & .00 & 00 & 00 & 00 & .00 & .00 & 00 & 0.00 \\
\hline 7 & 31 & 00 & 27 & 00 & 00 & 00 & 0 & 0 & 00 & 0 & 0 & 0 & 0 & .00 & 0 & 00 & 0 & 0.00 & 00 & 0 & 00 & 00 & .00 & .00 & 00 \\
\hline 08 & 39 & & 8 & 00 & 00 & 0 & & 0 & 00 & 0 & 0 & 00 & & & & 00 & 00 & 0.00 & 00 & 00 & 00 & .00 & .00 & 0.00 & .00 \\
\hline & 89 & 01 & & 00 & 00 & 00 & & & 0 & & & 0 & & & & 00 & 00 & 00 & 00 & 0 & 00 & 00 & .00 & 0.00 & م00 \\
\hline Z-10 & & & & & & & & & & & & & & & & & & & & & & & & & \\
\hline-11 & 13 & 01 & 57 & 00 & 00 & 00 & 00 & 00 & .00 & .00 & 00 & .00 & 00 & 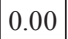 & 00 & 00 & .00 & .00 & .00 & 00 & .00 & .00 & .00 & 0.00 & .00 \\
\hline 12 & 55 & 01 & & 00 & 00 & 00 & & 0 & 0.00 & 00 & 0.00 & 0.00 & 00 & 0 & 0 & 0.00 & 0.00 & 0.00 & 00 & 00 & 00 & 0.00 & 0.00 & .00 & .00 \\
\hline 7_13 & 42 & 00 & 16 & 00 & 00 & 0 & & 0 & 0.00 & 0.00 & 0.00 & 0.00 & & & 00 & 0.00 & 0.00 & 0.00 & 0.00 & 00 & 00 & 0.00 & 0.00 & 0.00 & .00 \\
\hline Z-14 & 37 & & & 00 & م0 & & & & 0.00 & & 1 & & & & & 0.00 & 0.00 & 0.00 & 0.00 & 0 & 0 & 0.00 & 0 & 0 & 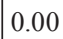 \\
\hline 210 & 17 & 50 & & 00 & 0 & 00 & & 0.00 & & 0.00 & 0.0 & 0.00 & & & & 0.00 & 0.0 & 0.0 & 0.00 & 0.00 & 0.00 & 0.00 & 0.00 & 0.00 &. \\
\hline Z-16 & 14 & & & & & & & & & & & & & & & & & 0 & & & & & & 0 & \\
\hline Z-17 & 13 & 24 & 20 & 00 & 00 & 00 & 00 & 0.00 & 0.00 & 0.00 & 0.00 & 0.00 & 00 & 0.00 & 0.00 & 0.00 & 0.00 & 0.00 & 0.00 & 0.00 & 0.00 & 0.00 & 0.00 & 0.00 & 0.00 \\
\hline Z-18 & 13 & 00 & 19 & 00 & 00 & 0 & 0 & 0 & 0.00 & 0.00 & 0.00 & 0.00 & 00 & 00 & 00 & 0.00 & 0.00 & 0.00 & 0.00 & 00 & 0.00 & 0.00 & 0.00 & 0.00 & 0.00 \\
\hline Z-19 & 25 & 00 & & 00 & 0.00 & 0.00 & & 0.00 & 0.00 & 0 & 0 & & & & & 0 & 0.00 & 0 & 0.00 & 0 & 0 & 0 & 0 & 0 & 0.00 \\
\hline $2-20$ & 46 & 01 & & 00 & 0.00 & 0 & 0.00 & 0.00 & 0.0 & 0.0 & 0.00 & 0.0 & & & 0.00 & 0.00 & 0.00 & 0.00 & 0.00 & .00 & 0.00 & 0.00 & 0.00 & 0.00 & 0.00 \\
\hline Z-21 & 0.58 & 0.01 & 0.08 & 0.00 & 0.00 & 0.00 & 0.00 & 0.00 & 0.00 & \begin{tabular}{|l|}
0.00 \\
\end{tabular} & \begin{tabular}{|l|}
0.00 \\
\end{tabular} & 0.00 & 0.00 & \begin{tabular}{|l|}
0.00 \\
\end{tabular} & 0.00 & 0.00 & 0.00 & \begin{tabular}{|l|}
0.00 \\
\end{tabular} & 0.00 & 0.00 & 0.00 & 0.00 & 0.00 & 0.00 & 0.00 \\
\hline 70 & 0.12 & 00 & ת م & 0.00 & 0.00 & 0.00 & 0.00 & 0.00 & 0.00 & 0.00 & 0.00 & 0.00 & 0.00 & 0 & 0.00 & 0.00 & 0 & 0.00 & 0.00 & 0.00 & 0.00 & 0.00 & 0.00 & 0.00 & 0.00 \\
\hline 7 & 42 & 00 & 17 & 00 & 00 & 0.00 & 0.00 & 0.00 & 0.00 & 00 & 00 & 00 & 0 & 0.00 & 0 & 00 & 0 & 0.00 & 0.00 & 0.00 & 0.00 & .00 & 00 & .00 & 0.00 \\
\hline 2 & 1.02 & 0.01 & 09 & 0.00 & 0.00 & 0.00 & 0.00 & 0.00 & 0.00 & 0.00 & 0.00 & 0.00 & 0.00 & 0.00 & 0.00 & 0.00 & 0.00 & 0.00 & 0.00 & 0.00 & 0.00 & 0.00 & 0.00 & 0.00 & 0.00 \\
\hline 25 & 0.00 & 0.01 & 0.59 & 0.00 & 0.00 & 0.00 & 0.00 & 0.00 & 0.00 & 0.00 & 0.00 & 0.00 & 0.00 & 0.00 & 0.00 & 0.00 & 0.00 & 0.00 & 0.00 & 0.00 & 0.00 & 0.00 & 0.00 & 0.00 & 0.00 \\
\hline
\end{tabular}




\section{Appendix C}
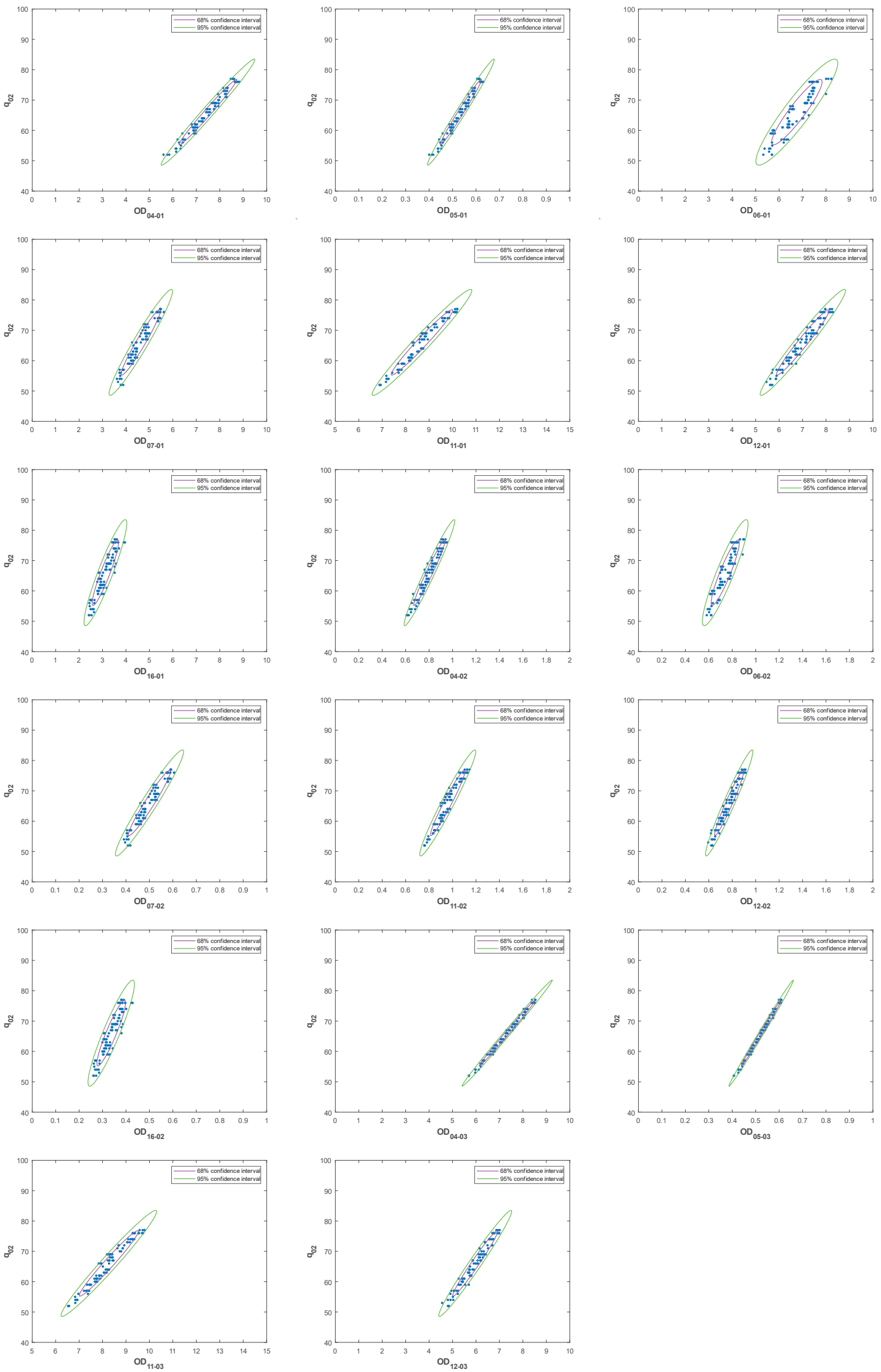

Fig. 10 Scatter-plots of the calibrated OD pairs $\left(O D^{c l b}\right)$ [vph] vs. the observed traffic flow $\left(q_{2}^{o b s}\right)[\mathrm{vph}]$ surrounded by $68 \%$ and $95 \%$ confidence intervals boundaries 


\section{Appendix D}
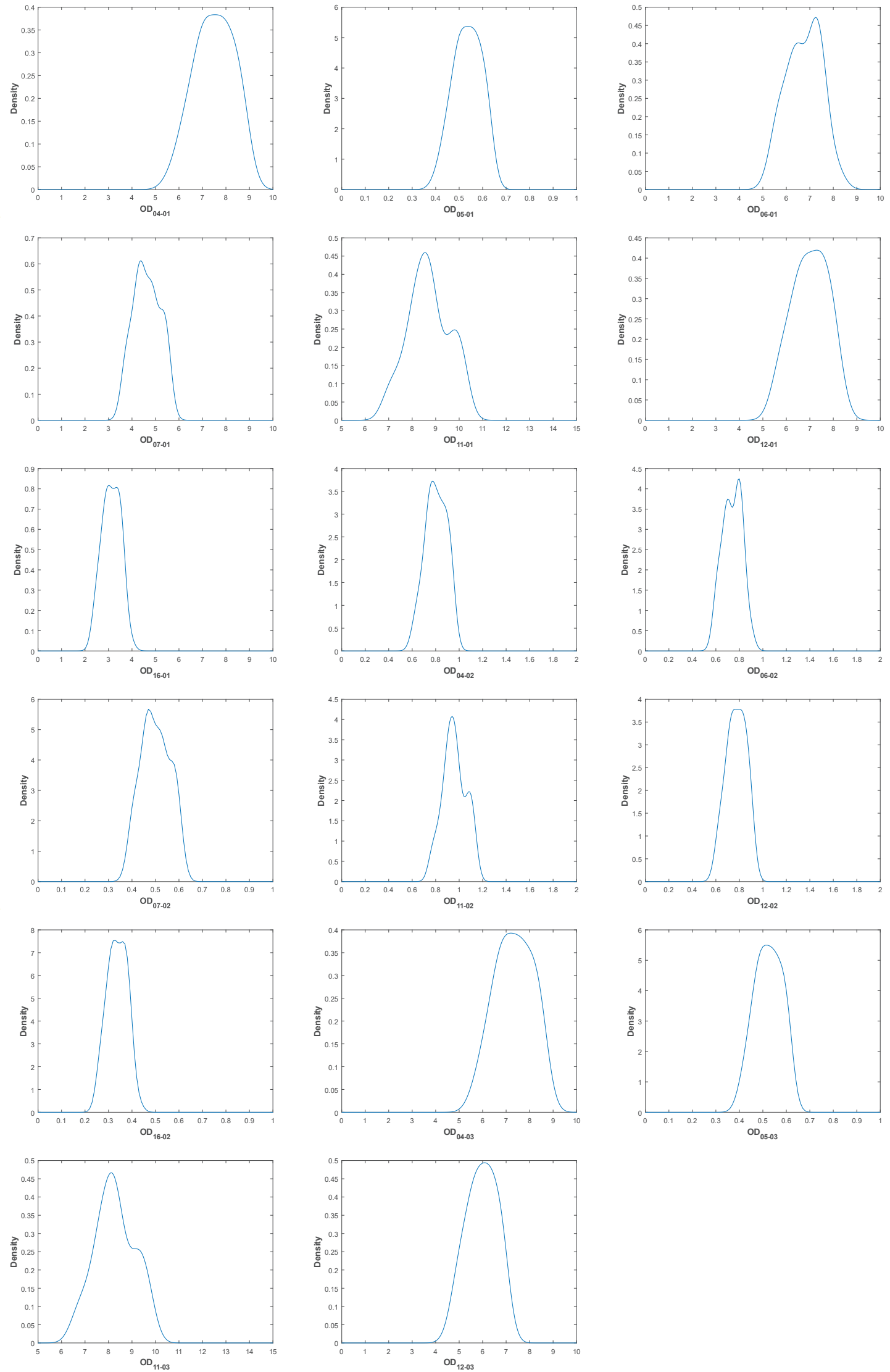

Fig. 11 Probability distributions of the OD pairs flows [vph] of the set $\mathcal{F}_{02}$ 